\title{
HOMOCLINIC POINTS, ATORAL POLYNOMIALS, AND PERIODIC POINTS OF ALGEBRAIC $\mathbb{Z}^{d}$-ACTIONS
}

\author{
DOUGLAS LIND, KLAUS SCHMIDT, AND EVGENY VERBITSKIY
}

\begin{abstract}
Cyclic algebraic $\mathbb{Z}^{d}$-actions are defined by ideals of Laurent polynomials in $d$ commuting variables. Such an action is expansive precisely when the complex variety of the ideal is disjoint from the multiplicative $d$-torus. For such expansive actions it is known that the limit for the growth rate of periodic points exists and is equal to the entropy of the action. In an earlier paper the authors extended this result to ideals whose variety intersects the $d$-torus in a finite set. Here we further extend it to the case when the dimension of intersection of the variety with the $d$-torus is at most $d-2$. The main tool is the construction of homoclinic points which decay rapidly enough to be summable.
\end{abstract}

\section{InTRODUCTION}

An algebraic $\mathbb{Z}^{d}$-action on a compact abelian group $X$ is a homomorphism $\alpha: \mathbb{Z}^{d} \rightarrow$ $\operatorname{aut}(X)$ from $\mathbb{Z}^{d}$ to the group of (continuous) automorphisms of $X$. We denote the image of $\mathbf{n} \in \mathbb{Z}^{d}$ under $\alpha$ by $\alpha^{\mathbf{n}}$, so that $\alpha^{\mathbf{m}+\mathbf{n}}=\alpha^{\mathbf{m}} \circ \alpha^{\mathbf{n}}$ and $\alpha^{\mathbf{0}}=\operatorname{Id}_{X}$.

We will consider here cyclic algebraic $\mathbb{Z}^{d}$-actions, described as follows, using notation and terminology from $[13]$. Let $R_{d}=\mathbb{Z}\left[u_{1}^{ \pm 1}, \ldots, u_{d}^{ \pm 1}\right]$ denote the ring of Laurent polynomials with integer coefficients in the commuting variables $u_{1}, \ldots, u_{d}$. We write $f \in R_{d}$ as $f=\sum_{\mathbf{m} \in \mathbb{Z}^{d}} f_{\mathbf{m}} \mathbf{u}^{\mathbf{m}}$, where $\mathbf{u}=\left(u_{1}, \ldots, u_{d}\right), \mathbf{m}=\left(m_{1}, \ldots, m_{d}\right) \in \mathbb{Z}^{d}$, $\mathbf{u}^{\mathbf{m}}=u_{1}^{m_{1}} \ldots u_{d}^{m_{d}}$, and $f_{\mathbf{m}} \in \mathbb{Z}$ with $f_{\mathbf{m}}=0$ for all but finitely many $\mathbf{m}$.

Let $\mathbb{T}=\mathbb{R} / \mathbb{Z}$, and define the shift $\mathbb{Z}^{d}$-action $\sigma$ on $\mathbb{T}^{\mathbb{Z}^{d}}$ by

$$
\left(\sigma^{\mathbf{m}} x\right)_{\mathbf{n}}=x_{\mathbf{m}+\mathbf{n}}
$$

for $\mathbf{m} \in \mathbb{Z}^{d}$ and $x=\left(x_{\mathbf{n}}\right) \in \mathbb{T}^{\mathbb{Z}^{d}}$. For $f=\sum f_{\mathbf{m}} \mathbf{u}^{\mathbf{m}} \in R_{d}$ we put

$$
f(\sigma)=\sum_{\mathbf{m} \in \mathbb{Z}^{d}} f_{\mathbf{m}} \sigma^{\mathbf{m}}: \mathbb{T}^{\mathbb{Z}^{d}} \rightarrow \mathbb{T}^{\mathbb{Z}^{d}}
$$

We identify $R_{d}$ with the dual group of $\mathbb{T}^{\mathbb{Z}^{d}}$ by setting

$$
\langle f, x\rangle=e^{2 \pi i f(\sigma)(x)_{\mathbf{0}}}=e^{2 \pi i \sum_{\mathbf{m}} f_{\mathbf{m}} x_{\mathbf{m}}}
$$

for $f \in R_{d}$ and $x \in \mathbb{T}^{\mathbb{Z}^{d}}$. In this identification the shift $\sigma^{\mathbf{m}}$ is dual to multiplication by $\mathbf{u}^{\mathbf{m}}$ on $R_{d}$. A closed subgroup $X \subset \mathbb{T}^{\mathbb{Z}^{d}}$ is shift-invariant if and only if its

Date: January 29, 2014.

2000 Mathematics Subject Classification. Primary: 37A35, 37B40, 54H20; Secondary: 37A45, 37D20, 13F20.

Key words and phrases. Entropy, periodic points, algebraic action. 
annihilator

$$
X^{\perp}=\left\{h \in R_{d}:\langle h, x\rangle=1 \text { for every } x \in X\right\}
$$

is an ideal in $R_{d}$. In view of this we write, for every ideal $\mathfrak{a}$ in $R_{d}$,

$$
X_{R_{d} / \mathfrak{a}}=\mathfrak{a}^{\perp}=\left\{x \in \mathbb{T}^{\mathbb{Z}^{d}}:\langle h, x\rangle=1 \text { for every } h \in \mathfrak{a}\right\}
$$

for the closed, shift-invariant subgroup of $\mathbb{T}^{\mathbb{Z}^{d}}$ annihilated by a. Here the dual group of $X_{R_{d} / \mathfrak{a}}$ is $R_{d} / \mathfrak{a}$. Denote by $\alpha_{R_{d} / \mathfrak{a}}$ the restriction of the shift-action $\sigma$ on $\mathbb{T}^{\mathbb{Z}^{d}}$ to $X_{R_{d} / \mathfrak{a}}$. A cyclic algebraic $\mathbb{Z}^{d}$-action is one of this form, corresponding to the cyclic $R_{d}$-module $R_{d} / \mathfrak{a}$.

According to [14, Eqn. (1-1)] or [18, Thm. 18.1], the topological entropy of $\alpha_{R_{d} / \mathfrak{a}}$, which coincides with its entropy with respect to Haar measure on $X_{R_{d} / \mathfrak{a}}$, is given by

$$
\mathrm{h}\left(\alpha_{R_{d} / \mathfrak{a}}\right)= \begin{cases}\infty & \text { if } \mathfrak{a}=\{0\} \\ \mathrm{m}(f) & \text { if } \mathfrak{a}=\langle f\rangle=f \cdot R_{d} \text { for some nonzero } f \in R_{d} \\ 0 & \text { if } \mathfrak{a} \text { is nonprincipal }\end{cases}
$$

where

$$
\mathrm{m}(f)=\int_{0}^{1} \ldots \int_{0}^{1} \log \left|f\left(e^{2 \pi i t_{1}}, \ldots, e^{2 \pi i t_{d}}\right)\right| d t_{1} \ldots d t_{d}
$$

is the logarithmic Mahler measure of $f$.

An algebraic $\mathbb{Z}^{d}$-action $\alpha$ on a compact abelian group $X$ is expansive if there is a neighborhood $U$ of $0_{X}$ such that $\bigcap_{\mathbf{m} \in \mathbb{Z}^{d}} \alpha^{\mathbf{m}}(U)=\left\{0_{X}\right\}$. To characterize expansiveness for cyclic actions $\alpha_{R_{d} / \mathfrak{a}}$, let $\mathbb{C}^{\times}$denote $\mathbb{C} \backslash\{0\}$ and let

$$
\mathrm{V}(\mathfrak{a})=\left\{\left(z_{1}, \ldots, z_{d}\right) \in\left(\mathbb{C}^{\times}\right)^{d}: g\left(z_{1}, \ldots, z_{d}\right)=0 \text { for all } g \in \mathfrak{a}\right\}
$$

denote the complex variety of the ideal $\mathfrak{a}$. Put $\mathbb{S}=\{z \in \mathbb{C}:|z|=1\}$, so that $\mathbb{S}^{d}$ is the unit multiplicative $d$-torus in $\left(\mathbb{C}^{\times}\right)^{d}$. Define the unitary variety of $\mathfrak{a}$ as

$$
\mathrm{U}(\mathfrak{a})=\mathrm{V}(\mathfrak{a}) \cap \mathbb{S}^{d}=\left\{\left(z_{1}, \ldots, z_{d}\right) \in \mathrm{V}(\mathfrak{a}):\left|z_{1}\right|=\cdots=\left|z_{d}\right|=1\right\} .
$$

According to [18, Thm. 6.5], $\alpha_{R_{d} / \mathfrak{a}}$ is expansive if and only if $\mathrm{U}(\mathfrak{a})=\varnothing$.

In order to describe periodic points for $\alpha_{R_{d} /\langle f\rangle}$, let $\mathcal{F}$ denote the collection of finite-index subgroups of $\mathbb{Z}^{d}$, and let $\Gamma$ be an arbitrary element of $\mathcal{F}$. Define

$$
\langle\Gamma\rangle=\min \{\|\mathbf{m}\|: \mathbf{0} \neq \mathbf{m} \in \Gamma\},
$$

where $\|\mathbf{m}\|=\max \left\{\left|m_{1}\right|, \ldots,\left|m_{d}\right|\right\}$. A point $x \in X$ has period $\Gamma$ if $\alpha^{\mathbf{m}} x=x$ for all $\mathbf{m} \in \Gamma$. Let

$$
\operatorname{Fix}_{\Gamma}\left(\alpha_{R_{d} / \mathfrak{a}}\right)=\left\{x \in X_{R_{d} / \mathfrak{a}}: x \text { has period } \Gamma\right\}
$$

be the closed subgroup of $X_{R_{d} / \mathfrak{a}}$ consisting of all $\Gamma$-periodic points. Since $\operatorname{Fix}_{\Gamma}\left(\alpha_{R_{d} / \mathfrak{a}}\right)$ may be infinite (examples are given in the next section), we reduce it to a finite object by forming the quotient of $\operatorname{Fix}_{\Gamma}\left(\alpha_{R_{d} / \mathfrak{a}}\right)$ by its connected component $\operatorname{Fix}_{\Gamma}^{\circ}\left(\alpha_{R_{d} / \mathfrak{a}}\right)$ of the identity. We therefore define

$$
\mathrm{P}_{\Gamma}\left(\alpha_{R_{d} / \mathfrak{a}}\right)=\left|\operatorname{Fix}_{\Gamma}\left(\alpha_{R_{d} / \mathfrak{a}}\right) / \operatorname{Fix}_{\Gamma}^{\circ}\left(\alpha_{R_{d} / \mathfrak{a}}\right)\right|,
$$

where $|\cdot|$ denotes cardinality. 
Define the upper logarithmic growth rate of the number of periodic components of $\alpha_{R_{d} / \mathfrak{a}}$ as

$$
\mathrm{p}^{+}\left(\alpha_{R_{d} / \mathfrak{a}}\right):=\limsup _{\langle\Gamma\rangle \rightarrow \infty} \frac{1}{\left|\mathbb{Z}^{d} / \Gamma\right|} \log \mathrm{P}_{\Gamma}\left(\alpha_{R_{d} / \mathfrak{a}}\right)
$$

For an arbitrary nonzero ideal $\mathfrak{a}$ it was proved in [18, Thm. 21.1] that $\mathrm{p}^{+}\left(\alpha_{R_{d} / \mathfrak{a}}\right)=$ $\mathrm{h}\left(\alpha_{R_{d} / \mathfrak{a}}\right)$. For expansive actions much more is known.

Theorem 1.1. Let $\mathfrak{a}$ be an ideal for which $\alpha_{R_{d} / \mathfrak{a}}$ is expansive, or equivalently for which $\mathrm{U}(\mathfrak{a})=\varnothing$. The the limsup in (1.2) is actually a limit, i.e.,

$$
\lim _{\langle\Gamma\rangle \rightarrow \infty} \frac{1}{\left|\mathbb{Z}^{d} / \Gamma\right|} \log \mathrm{P}_{\Gamma}\left(\alpha_{R_{d} / \mathfrak{a}}\right)=\mathrm{h}\left(\alpha_{R_{d} / \mathfrak{a}}\right) .
$$

It is not known whether (1.3) holds for all cyclic actions with nonzero $\mathfrak{a}$ (when $\mathfrak{a}=0$ the left side is 0 and the right side is $\infty$ ). Even when $d=1$ the existence of this limit involves a deep diophantine estimate due to Gelfond [9] (see Section 7, or [11, Sec. 4] for details). In [13] we proved the following partial result.

Theorem 1.2. Let $d \geqslant 2$ and $\mathfrak{a}$ be an ideal in $R_{d}$ whose unitary variety $\mathrm{U}(\mathfrak{a})$ is a finite set. Then (1.3) holds.

The proof of Theorem 1.2 in [13] depends on the crucial fact that if $U(\mathfrak{a})$ is finite, then all of its points have coordinates which are algebraic numbers. With this information Theorem 1.2 can be deduced from Gelfond's estimate. However, the route taken in [13] is different, and bypasses the diophantine issue: the algebraicity of the points in $\mathrm{U}(\mathfrak{a})$ allows the construction of certain well-behaved (so-called summable) homoclinic points of $\alpha_{R_{d} / \mathfrak{a}}$, which are then used to prove not only Theorem 1.2 but also a strong specification property of the action $\alpha_{R_{d} / \mathfrak{a}}$ which is of independent interest. We remark in passing that this approach involving summable homoclinic points first appeared in [19] in the construction of symbolic covers for a special class of cyclic $\mathbb{Z}^{d}$-actions, the harmonic actions.

In order to state our main result, we need to recall some notions from real algebraic geometry. Let $z_{j}=x_{j}+i y_{j}$, and consider $\mathbb{C}^{d}$ as $\mathbb{R}^{2 d}$ with coordinates $\left(x_{1}, y_{1}, \ldots, x_{d}, y_{d}\right)$. Then $\mathbb{S}^{d}$ as a subset of $\mathbb{R}^{2 d}$ is the real algebraic set defined by the equations $x_{j}^{2}+y_{j}^{2}-1=0$ for $1 \leqslant j \leqslant d$. For $f\left(z_{1}, \ldots, z_{d}\right) \in \mathbb{C}\left[z_{1}, \ldots, z_{d}\right]$, we can expand $f$ into its real an imaginary polynomial parts, so that

$$
f\left(x_{1}+i y_{1}, \ldots, x_{d}+i y_{d}\right)=f_{1}\left(x_{1}, y_{1}, \ldots, x_{d}, y_{d}\right)+i f_{2}\left(x_{1}, y_{1}, \ldots, x_{d}, y_{d}\right),
$$

where $f_{1}, f_{2} \in \mathbb{R}\left[x_{1}, y_{1}, \ldots, x_{d}, y_{d}\right]$ have real coefficients. Then $\mathbf{U}(f)$ is the real algebraic set defined by the equations $x_{j}^{2}+y_{j}^{2}-1=0$ for $1 \leqslant j \leqslant d$ together with two further equations $f_{1}\left(x_{1}, y_{1}, \ldots, x_{d}, y_{d}\right)=0$ and $f_{2}\left(x_{1}, y_{1}, \ldots, x_{d}, y_{d}\right)=0$. This discussion extends to Laurent polynomials $f \in R_{d}$ by observing that there is a $\mathbf{m} \in$ $\mathbb{Z}^{d}$ with $\mathbf{u}^{\mathbf{m}} f(\mathbf{u}) \in \mathbb{Z}\left[u_{1}, \ldots, u_{d}\right]$ and that $\mathbf{U}\left(\mathbf{u}^{\mathbf{m}} f\right)=\mathbf{U}(f)$. Every ideal $\mathfrak{a}$ is finitely generated, say by $g_{1}, \ldots, g_{r}$. Hence its unitary variety $\mathbf{U}(\mathfrak{a})=\mathbf{U}\left(g_{1}\right) \cap \cdots \cap \mathbf{U}\left(g_{r}\right)$ is again a real algebraic subset of $\mathbb{R}^{2 d}$. 
The cell decomposition theorem for real semialgebraic sets [7, Thm. 2.11] therefore applies, and so $U(\mathfrak{a})$ can be written as a finite disjoint union of open cells of various dimensions. The dimension of $\mathrm{U}(\mathfrak{a})$ is defined to be the maximum dimension of these cells, and this number is the same for all possible cell decompositions. By convention we define $\operatorname{dim} \varnothing=-\infty$. There is a finite algorithm [3, Algorithm 14.10] for computing the dimension of a real semialgebraic set defined by polynomials with rational coefficients.

Our main result is the following extension of Theorem 1.2 .

Theorem 1.3. Let $d \geqslant 2$ and let $\mathfrak{a}$ be an ideal in $R_{d}$. If the dimension of $\mathrm{U}(\mathfrak{a})$ is at most $d-2$, then

$$
\lim _{\langle\Gamma\rangle \rightarrow \infty} \frac{1}{\left|\mathbb{Z}^{d} / \Gamma\right|} \log \mathrm{P}_{\Gamma}\left(\alpha_{R_{d} / \mathfrak{a}}\right)=\mathrm{h}\left(\alpha_{R_{d} / \mathfrak{a}}\right) .
$$

In [13] it is explained how to use the algebraic machinery in [18, Sec. 21] to reduce the proof of Theorem 1.3 to the case when $\mathfrak{a}$ is prime and principal. In view of this we assume from now on that $\mathfrak{a}=(f)=f R_{d}$ for some nonzero irreducible Laurent polynomial $f \in R_{d}$. In this case we call the shift-action $\alpha_{f}:=\alpha_{R_{d} /\langle f\rangle}$ on the group $X_{f}:=X_{R_{d} /(f)}$ a principal algebraic $\mathbb{Z}^{d}$-action.

Let $\Omega$ denote the set of torsion points in $\mathbb{S}^{d}$, so the coordinates of points in $\Omega$ are roots of unity. For $\Gamma \in \mathcal{F}$ let

$$
\Omega_{\Gamma}:=\left\{\boldsymbol{\omega} \in \Omega: \boldsymbol{\omega}^{\mathbf{m}}=\omega_{1}^{m_{1}} \cdots \omega_{d}^{m_{d}}=1 \text { for every } \mathbf{m} \in \Gamma\right\} .
$$

Basic duality shows that $\Omega_{\Gamma}$ is the dual group of $\mathbb{Z}^{d} / \Gamma$, and so $\left|\Omega_{\Gamma}\right|=\left|\mathbb{Z}^{d} / \Gamma\right|$.

As mentioned above, according to [18, Thm. 21.1],

$$
\mathrm{p}^{+}\left(\alpha_{f}\right):=\limsup _{\langle\Gamma\rangle \rightarrow \infty} \frac{1}{\left|\mathbb{Z}^{d} / \Gamma\right|} \log \mathrm{P}_{\Gamma}\left(\alpha_{f}\right)=\mathrm{h}\left(\alpha_{f}\right)=\mathrm{m}(f)=\int_{\mathbb{S}^{d}} \log |f(\mathbf{s})| d \lambda(\mathbf{s}),
$$

where $\lambda$ is normalized Lebesgue measure on $\mathbb{S}^{d}$. In [13, Lemma 2.1] we claimed that

$$
\mathrm{P}_{\Gamma}\left(\alpha_{f}\right)=\prod_{\boldsymbol{\omega} \in \Omega_{\Gamma} \backslash \mathrm{U}(f)}|f(\boldsymbol{\omega})| .
$$

However, using the notation from the proof there, we in fact need to divide the right-hand side of (1.5) by

$$
c_{\Gamma}(f)=\left|f(\widetilde{\sigma})\left(V_{\Gamma}(\mathbb{Z})\right) / f(\widetilde{\sigma})\left(V_{\Gamma}^{\prime}(\mathbb{Z})\right)\right| .
$$

The proofs of the main results in both [13] and here do not depend on (1.5), and show that

$$
\frac{1}{\left|\mathbb{Z}^{d} / \Gamma\right|} \log c_{\Gamma}(f) \rightarrow 0 \quad \text { as } \quad\langle\Gamma\rangle \rightarrow \infty
$$

so that (1.5) is asymptotically correct.

Observe that since $\left|\mathbb{Z}^{d} / \Gamma\right|=\left|\Omega_{\Gamma}\right|$, we are dealing with sums of the form

$$
\frac{1}{\left|\Omega_{\Gamma}\right|} \sum_{\boldsymbol{\omega} \in \Omega_{\Gamma} \backslash \mathrm{U}(f)} \log |f(\boldsymbol{\omega})|,
$$


which are Riemann sum approximations to $\int_{\mathbb{S}^{d}} \log |f| d \lambda=\mathrm{m}(f)$. Hence proving the existence of the limit in Theorem 1.3 is exactly the same as proving that these Riemann sums for $\log |f|$ converge to its integral over $\mathbb{S}^{d}$ as $\langle\Gamma\rangle \rightarrow \infty$. In trying to prove convergence of these Riemann sums one encounters two problems.

The first problem involves the omission of summands in (1.6) with $\boldsymbol{\omega} \in \Omega_{\Gamma} \cap \mathrm{U}(f)$. As we will see, each such $\boldsymbol{\omega}$ contributes one dimension to $\operatorname{Fix}_{\Gamma}^{\circ}\left(\alpha_{f}\right)$, so that $\operatorname{Fix}_{\Gamma}^{\circ}\left(\alpha_{f}\right)$ is a torus of dimension $\left|\Omega_{\Gamma} \cap \mathrm{U}(f)\right|$. This omission is necessary, of course, since any summand with $f(\boldsymbol{\omega})=0$ would contribute $-\infty$ to the Riemann sum. This situation was easily dealt with in the case $\mathrm{U}(f)$ is finite [13] by observing that the dimension of this torus is then bounded, so there is an easy bound on the number of points in any separated set of any closed subgroup. However, here the dimension of this torus can be unbounded (see Example 4.6). We control this by invoking a result of Mann [15] that implies that all torsion points in $\mathrm{U}(f)$ lie in a finite union of cosets of rational subtori, and this provides a sufficiently uniform estimate for separated sets.

The second problem involves those $\boldsymbol{\omega} \in \Omega_{\Gamma} \backslash \mathrm{U}(f)$ which may be very close to $\mathrm{U}(f)$, and is much more serious. For these points the value $|f(\boldsymbol{\omega})|$ will be extremely small, but nonzero, and so $\log |f(\boldsymbol{\omega})|$ could conceivably take such a large negative value that the average value in (1.6) is significantly less than $\mathrm{m}(f)$. Can this happen for a sequence of $\Gamma_{n}$ with $\left\langle\Gamma_{n}\right\rangle \rightarrow \infty$ ? This problem is essentially a multidimensional version of the diophantine problem mentioned earlier.

Unfortunately this diophantine problem is unsolved in the generality required here, which forces us to impose the additional hypothesis that $\mathrm{U}(f)$ has dimension at most $d-2$. This hypothesis turns out to be equivalent to the existence of summable homoclinic points (Theorem 3.2), and allows us to extend the approach developed in [19] and [13] to this more general situation, thereby proving Theorem 1.3. As a side benefit of this approach, we obtain in Corollary 7.3 a diophantine estimate concerning the proximity of torsion points to the variety $\mathrm{U}(f)$ under the hypothesis that $\operatorname{dim} \mathrm{U}(f) \leqslant d-2$. This result is completely analogous to Gelfond's estimate. In the case when $\operatorname{dim} \mathrm{U}(f)=d-1$ (which when $d=1$ reduces to the setting of Gelfond's estimate), our approach yields a slightly weaker estimate (Corollary 7.5).

The question of whether entropy coincides with the logarithmic growth rate of the number of connected components in $\operatorname{Fix}_{\Gamma}\left(\alpha_{f}\right)$ as $\langle\Gamma\rangle \rightarrow \infty$ irrespective of whether the principal action is expansive or not is a special case of a much more general problem. For a principal algebraic $\mathbb{Z}^{d}$-action $\alpha_{f}$ the entropy was proved in [14] to be equal to the logarithmic Mahler measure of the Laurent polynomial $f \in R_{d}$, and to the upper logarithmic growth rate of $\mathrm{P}_{\Gamma}\left(\alpha_{f}\right)$ in [18. For an expansive principal algebraic action $\alpha_{f}$ of an arbitrary residually finite countable amenable group $G$, the entropy $\mathrm{h}\left(\alpha_{f}\right)$ was identified in [6] with an appropriately defined logarithmic growth rate of the number of points with finite orbits of $\alpha_{f}$ and, as a consequence, with the Fuglede-Kadison determinant associated with the element $f$ in the integral group ring $\mathbb{Z}[G]$, acting on the group von Neumann algebra $\mathcal{N}[G]$. Without the hypothesis of expansiveness, the relationship between entropy, logarithmic growth rate of periodic points (or periodic components), and Fuglede-Kadison determinants 
is still completely open. In this sense, the equality of $\mathrm{p}^{+}\left(\alpha_{f}\right)$ and $\mathrm{h}\left(\alpha_{f}\right)$, and the more precise version in Theorem 1.3 are of interest, since they provide links between these objects without the hypothesis of expansiveness (but obviously under very special circumstances).

The authors are grateful to the Erwin Schrödinger International Institute for Mathematical Physics, the Max Planck Institute for Mathematics, FWF Grant S9613, the University of Washington Mathematics Department, the Lorentz Center in Leiden, and Microsoft Research for their generous support of this work.

\section{Atoral polynomials}

Here we characterize those polynomials for which our techniques apply. Recall that the units of $R_{d}$ are $\pm \mathbf{u}^{\mathbf{n}}$, where $\mathbf{n}$ is an arbitrary element of $\mathbb{Z}^{d}$. We say that a Laurent polynomial in $R_{d}$ is irreducible if it is not a unit and if it has no factorizations apart from units.

The basic notion of atorality is motivated by the paper of Agler, McCarthy, and Stankus [1].

Definition 2.1. An irreducible Laurent polynomial $f \in R_{d}$ is called toral if $h$ is in the ideal $(f)$ whenever $h$ is in $R_{d}$ with $\mathrm{U}(h) \supseteq \mathrm{U}(f)$. Otherwise $f$ is called atoral. A general Laurent polynomial is called atoral if each of its irreducible factors is atoral; otherwise it is called toral.

In [1] the notion of atorality of polynomials with complex coefficients is introduced and studied. One main result there is that such a polynomial is atoral if and only if its unitary variety is contained in an algebraic set of (complex) dimension $d-2$. Our setting, involving polynomials with rational coefficients and a corresponding notion of irreducibility, is somewhat different. However, we obtain a similar result, whose proof follows closely the spirit of [1].

Proposition 2.2. Let $f \in R_{d}$ be irreducible. Then $f$ is atoral if and only if $\operatorname{dim} U(f) \leqslant d-2$.

Symmetry plays a crucial role in the proof.

Definition 2.3. Let $f(\mathbf{u})=\sum f_{\mathbf{n}} \mathbf{u}^{\mathbf{n}} \in R_{d}$. The adjoint of $f$ is $f^{*}(\mathbf{u})=f\left(\mathbf{u}^{-1}\right)=$ $\sum f_{-\mathbf{n}} \mathbf{u}^{\mathbf{n}}$. We say that $f$ is symmetric if $f^{*}(\mathbf{u})=f(\mathbf{u})$, and that $f$ is essentially symmetric if there is an $\mathbf{m} \in \mathbb{Z}^{d}$ such that $f^{*}(\mathbf{u})= \pm \mathbf{u}^{\mathbf{m}} f(\mathbf{u})$.

Thus $f$ is symmetric if its array of coefficients is symmetric with respect to the origin, and is essentially symmetric if this array is symmetric (or skew-symmetric) with respect to the point $\frac{1}{2} \mathbf{m} \in \mathbb{R}^{d}$.

Lemma 2.4. Let $f \in R_{d}$. Then $f$ is essentially symmetric if and only if $f^{*} \in(f)$.

Proof. If $f$ is essentially symmetric, then obviously $f^{*}(\mathbf{u})= \pm \mathbf{u}^{\mathbf{m}} f(\mathbf{u}) \in(f)$. For the opposite implication we may assume that $f \neq 0$. Suppose that $f^{*}=h f$ for some $h \in R_{d}$. Then $f=h^{*} f^{*}=h^{*} h f$, so that $\left(h^{*} h-1\right) f=0$. Since $f \neq 0$, we obtain that $h^{*} h=1$. But the only units in $R_{d}$ are $\pm \mathbf{u}^{\mathbf{m}}$. 
Lemma 2.5. Let $f \in R_{d}$. Then $\mathrm{U}\left(f^{*}\right)=\mathrm{U}(f)$.

Proof. Since $f$ has real coefficients, for every $\mathbf{s} \in \mathbb{S}^{d}$ we have that $f^{*}(\mathbf{s})=\overline{f(\mathbf{s})}$.

Lemma 2.6. Let $r \geqslant 2$ and suppose that $g_{1}, \ldots, g_{r} \in \mathbb{Q}\left[u_{1}, \ldots, u_{d}\right]$ have no common factor. Let $\mathfrak{g}$ be the ideal in $\mathbb{Q}\left[u_{1}, \ldots, u_{d}\right]$ they generate. Then $\operatorname{dim} \mathrm{U}(\mathfrak{g}) \leqslant d-2$.

Proof. We use induction on $d$. First suppose that $d=1$, and that $g_{1}, \ldots, g_{r} \in \mathbb{Q}\left[u_{1}\right]$ have no common factor. Then their greatest common divisor is 1 , so there are $A_{j}\left(u_{1}\right) \in \mathbb{Q}\left[u_{1}\right]$ such that $\sum_{j=1}^{r} A_{j}\left(u_{1}\right) g_{j}\left(u_{1}\right)=1$. Hence $\mathbf{U}(\mathfrak{g})=\bigcap_{j=1}^{r} \mathrm{U}\left(g_{j}\right)=\varnothing$, and $\operatorname{dim} \varnothing=-\infty \leqslant-1$.

To complete the induction argument, assume that the result is true for $d-1$, and let $g_{1}, \ldots, g_{r} \in \mathbb{Q}\left[u_{1}, \ldots, u_{d}\right]$ have no common factor. Put $K=\mathbb{Q}\left(u_{1}, \ldots, u_{d-1}\right)$ and consider

$$
g_{j}\left(u_{1}, \ldots, u_{d}\right)=\sum_{k} g_{j k}\left(u_{1}, \ldots, u_{d-1}\right) u_{d}^{k} \in K\left[u_{d}\right] .
$$

By Gauss's Lemma, the greatest common divisor of the $g_{j}$ as elements in $K\left[u_{d}\right]$ is 1 , so there are

$$
a_{j}\left(u_{1}, \ldots, u_{d}\right)=\sum_{k} a_{j k}\left(u_{1}, \ldots, u_{d-1}\right) u_{d}^{k}
$$

with $a_{j k}\left(u_{1}, \ldots, u_{d-1}\right) \in \mathbb{Q}\left(u_{1}, \ldots, u_{d-1}\right)$ such that

$$
\sum_{j=1}^{r} a_{j}\left(u_{1}, \ldots, u_{d}\right) g_{j}\left(u_{1}, \ldots, u_{d}\right)=1 .
$$

Clearing denominators, we obtain

$$
A_{j}\left(u_{1}, \ldots, u_{d}\right) \in \mathbb{Q}\left[u_{1}, \ldots, u_{d}\right] \quad \text { and } \quad 0 \neq B\left(u_{1}, \ldots, u_{d-1}\right) \in \mathbb{Q}\left[u_{1}, \ldots, u_{d-1}\right]
$$

such that

$$
\sum_{j=1}^{r} A_{j}\left(u_{1}, \ldots, u_{d}\right) g_{j}\left(u_{1}, \ldots, u_{d}\right)=B\left(u_{1}, \ldots, u_{d-1}\right) .
$$

Define $\pi: \mathbb{S}^{d} \rightarrow \mathbb{S}^{d-1}$ by $\pi\left(s_{1}, \ldots, s_{d}\right)=\left(s_{1}, \ldots, s_{d-1}\right)$. Since $B \neq 0$, it follows that $\operatorname{dim} \mathrm{U}(B) \leqslant d-2$ as a subset of $\mathbb{S}^{d-1}$. Let $Y=\left\{\mathbf{s} \in \mathbf{U}(B): g_{j k}(\mathbf{s}) \neq 0\right.$ for some $\left.j, k\right\}$ and $Z=\left\{\mathbf{s} \in \mathrm{U}(B): g_{j k}(\mathbf{s})=0\right.$ for all $\left.j, k\right\}$. If $\mathbf{s} \in Y$ then some $g_{j}\left(\mathbf{s}, u_{d}\right) \neq 0$, so that $\pi^{-1}(\mathbf{s}) \cap Y$ is finite. Hence $\operatorname{dim}\left(\pi^{-1}(Y) \cap \mathrm{U}(\mathfrak{g})\right) \leqslant \operatorname{dim} \mathrm{U}(B) \leqslant d-2$. Observe that the $g_{j k}\left(u_{1}, \ldots, u_{d-1}\right)$ cannot have a common factor in $\mathbb{Q}\left[u_{1}, \ldots, u_{d-1}\right]$, since this would contradict our assumption on the $g_{j}$ 's. Hence by the inductive hypothesis applied to the $g_{j k}$ 's, we find that $Z$, being the unitary variety of the ideal generated by the $g_{j k}$, has dimension at most $d-3$. Hence $\operatorname{dim} \pi^{-1}(Z) \cap \mathrm{U}(\mathfrak{g}) \leqslant d-2$. Thus $\mathrm{U}(\mathfrak{g})$ is the union of the two semialgebraic sets $\pi^{-1}(Y) \cap \mathrm{U}(\mathfrak{g})$ and $\pi^{-1}(Z) \cap \mathrm{U}(\mathfrak{g})$, each of which has dimension $\leqslant d-2$, completing the induction step, and the proof.

Proof of Proposition 2.2. First suppose that $f \in R_{d}$ is irreducible and atoral. Thus there is a $g \in R_{d} \backslash(f)$ with $\mathrm{U}(g) \supseteq \mathrm{U}(f)$. Now $f$ and $g$ cannot have a common factor since $f$ does not divide $g$, so that by Lemma 2.6 applied to $g_{1}=f$ and $g_{2}=g$, we obtain that $\mathrm{U}(f)=\mathrm{U}(f) \cap \mathrm{U}(g)$ has dimension $\leqslant d-2$. 
For the reverse implication, suppose that $f \in R_{d}$ is irreducible and that $\operatorname{dim} \mathrm{U}(f) \leqslant$ $d-2$.

If $f$ is not essentially symmetric, then $g=f^{*} \notin(f)$ by Lemma 2.4 and $\mathrm{U}(g)=$ $\mathrm{U}(f)$ by Lemma 2.5, hence $f$ is atoral.

Suppose now that $f$ is essentially symmetric, and that $f^{*}(\mathbf{u})=\mathbf{u}^{\mathbf{m}} f(\mathbf{u})$. Observe that if $f_{2}\left(u_{1}, \ldots, u_{d}\right)=f\left(u_{1}^{2}, \ldots, u_{d}^{2}\right)$, then $\mathrm{U}\left(f_{2}\right)$ is the union of $2^{d}$ smaller copies of $\mathbf{U}(f)$ and that $f_{2}^{*}(\mathbf{u})=\mathbf{u}^{2 \mathbf{m}} f_{2}(\mathbf{u})$. Hence by replacing $f$ with $f_{2}$ we may assume that $\mathbf{m}=2 \mathbf{n} \in 2 \mathbb{Z}^{d}$. Furthermore, replacing $f(\mathbf{u})$ with $\mathbf{u}^{\mathbf{n}} f(\mathbf{u})$ (which preserves unitary varieties), we may assume that $f^{*}=f$ is exactly symmetric.

We next show that the partial derivatives of $f$ must also vanish on $\mathrm{U}(f)$. Symmetry of $f$ means that $f$ is real-valued on $\mathbb{S}^{d}$. Let $e: \mathbb{T}^{d} \rightarrow \mathbb{S}^{d}$ be the isomorphism $e\left(t_{1}, \ldots, t_{d}\right)=\left(e^{2 \pi i t_{1}}, \ldots, e^{2 \pi i t_{d}}\right)$. Then $f \circ e: \mathbb{T}^{d} \rightarrow \mathbb{S}^{d}$ vanishes on $e^{-1}(\mathrm{U}(f))$. Let $\mathbf{s} \in \mathrm{U}(f)$ and suppose that there is a $j$ for which $\partial f / \partial u_{j}(\mathbf{s}) \neq 0$. By the chain rule, $\partial(f \circ e) / \partial t_{j}\left(e^{-1}(\mathbf{s})\right) \neq 0$, hence the gradient of $f \circ e$ does not vanish at $e^{-1}(\mathbf{s})$. The Implicit Function Theorem shows that near $e^{-1}(\mathbf{s})$ the vanishing set of $f \circ e$ is $(d-1)$-dimensional, and hence $\mathrm{U}(f)$ is $(d-1)$-dimensional, contradicting our assumption.

Hence all partials $\partial f / \partial u_{j}$ vanish on $\mathrm{U}(f)$. The case of constant $f$ is trivial, so we may assume that at least one $\partial f / \partial u_{j} \neq 0$. But the $u_{j}$ degree of this partial is strictly less than the $u_{j}$-degree of $f$, and so cannot have a factor in common with $f$ by irreducibility of $f$. Hence using $g=\partial f / \partial u_{j}$ shows that $f$ is atoral.

Finally, if $f$ is essentially symmetric with $f^{*}(\mathbf{u})=-\mathbf{u}^{\mathbf{m}} f(\mathbf{u})$, our previous simplifications show that we may assume that $f^{*}=-f$. But then $f$ is purely imaginary on $\mathbb{S}^{d}$, so we can apply the preceding argument to $f / i$ and again obtain that $f$ is atoral.

Remark 2.7. Since $\mathbf{U}\left(f_{1} \cdots f_{r}\right)=\mathbf{U}\left(f_{1}\right) \cup \cdots \cup \mathbf{U}\left(f_{r}\right)$, and since the dimension of the union of a finite number of semialgebraic sets equals the largest of the dimensions of those sets, it follows that Proposition 2.2 remains true for all $f \in R_{d}$.

\section{Summable HOMOCLINIC POINTS}

Let $\alpha$ be an algebraic $\mathbb{Z}^{d}$-action on a compact abelian group $X$. We recall from [12] that a point $x \in X$ is homoclinic for $\alpha$ if $\alpha^{\mathbf{n}}(x) \rightarrow 0$ as $\|\mathbf{n}\| \rightarrow \infty$. The set of all homoclinic points in $X$ is a subgroup that we denote by $\Delta_{\alpha}(X)$. If $\alpha$ is expansive then the homoclinic group $\Delta_{\alpha}(X)$ is countable, and additionally $\alpha^{\mathbf{n}}(x) \rightarrow$ 0 exponentially fast as $\|\mathbf{n}\| \rightarrow \infty$ for every $x \in \Delta_{\alpha}(X)$. If $\alpha$ is nonexpansive then $\Delta_{\alpha}(X)$ may be uncountable, countable, or trivial, and $\alpha$-homoclinic points may decay very slowly (see [12] for details, examples, and connections with entropy).

For the proof of Theorem 1.3 we need homoclinic points which decay sufficiently rapidly. To describe our requirements more precisely, we write $\|t\|$ for the distance from a point $t \in \mathbb{T}$ to 0 . A point $x \in X_{f}$ is called a summable homoclinic point for $\alpha_{f}$ if $\sum_{\mathbf{n} \in \mathbb{Z}^{d}}\left\|x_{\mathbf{n}}\right\|<\infty$. Denote the subgroup of summable homoclinic points by $\Delta_{\alpha_{f}}^{1}\left(X_{f}\right)$, which is obviously a subgroup of $\Delta_{\alpha_{f}}\left(X_{f}\right)$. 
If $\alpha_{f}$ is expansive then $\mathrm{U}(f)=\varnothing$, and so $1 / f^{*}$ is analytic on $\mathbb{S}^{d}$. As shown in [12], the Fourier coefficients of $1 / f^{*}$ provide a nonzero homoclinic point for $\alpha_{f}$ that decays exponentially fast, hence is summable. When $\mathrm{U}(f) \neq \varnothing$, the same approach will work provided there is a $g \in R_{d} \backslash(f)$ for which $g / f^{*}$ is smooth enough to have absolutely convergent Fourier series. The existence of such a $g$ exactly depends on whether or not $f$ is atoral.

We begin with some Fourier machinery. For $a=\left(a_{\mathbf{n}}\right) \in \ell^{1}\left(\mathbb{Z}^{d}, \mathbb{C}\right)$ define its Fourier transform $\widehat{a}: \mathbb{S}^{d} \rightarrow \mathbb{C}$ by $\widehat{a}(\mathbf{s})=\sum_{\mathbf{n} \in \mathbb{Z}^{d}} a_{\mathbf{n}} \mathbf{s}^{\mathbf{n}}$, where $\mathbf{s}^{\mathbf{n}}=s_{1}^{n_{1}} \cdots s_{d}^{n_{d}}$. If $\phi: \mathbb{S}^{d} \rightarrow \mathbb{C}$ is integrable with respect to Haar measure $\lambda$ on $\mathbb{S}^{d}$, we define its Fourier coefficients by $\widehat{\phi}_{\mathbf{n}}=\int \phi(\mathbf{s}) \mathbf{s}^{-\mathbf{n}} d \lambda(\mathbf{s})$. A polynomial $g=\sum g_{\mathbf{n}} \mathbf{u}^{\mathbf{n}} \in R_{d}$ can be considered as an element in $\ell^{1}\left(\mathbb{Z}^{d}, \mathbb{C}\right)$, and as such $\widehat{g}$ corresponds to the polynomial function on $\mathbb{S}^{d}$.

We return to our given nonzero irreducible polynomial $f \in R_{d} \subset \ell^{1}\left(\mathbb{Z}^{d}, \mathbb{C}\right)$. Define ideals $\mathfrak{n}_{f}$ and $\mathfrak{m}_{f}$ of $R_{d}$ by

$$
\begin{gathered}
\mathfrak{n}_{f}=\left\{h \in R_{d}:\left.h\right|_{\mathrm{U}(f)} \equiv 0\right\}, \\
\mathfrak{m}_{f}=\left\{h \in R_{d}: \widehat{h} / \widehat{f} \text { has absolutely convergent Fourier series }\right\} .
\end{gathered}
$$

Clearly $(f) \subseteq \mathfrak{m}_{f} \subseteq \mathfrak{n}_{f}$. By definition, $f$ is toral if and only if these ideals coincide.

Lemma 3.1. Let $\sqrt{\mathfrak{m}_{f}}$ denote the radical ideal of $\mathfrak{m}_{f}$. Then $\sqrt{\mathfrak{m}_{f}}=\mathfrak{n}_{f}$.

Proof. If $g \in \sqrt{\mathfrak{m}_{f}}$, then $g$ (or, more precisely, the Fourier transform $\widehat{g}: \mathbb{S}^{d} \rightarrow \mathbb{C}$ ) must vanish on $\mathrm{U}(f)$. Hence $\sqrt{\mathfrak{m}_{f}} \subseteq \mathfrak{n}_{f}$.

For the reverse inclusion we use the Lojasiewicz inequality from real algebraic geometry. Recall that we can consider $\mathbb{C}^{d}=\mathbb{R}^{2 d}$ with coordinates $x_{1}, y_{1}, \ldots, x_{d}, y_{d}$. We may assume that $f \in R_{d}$ is a polynomial, and expand $f=f_{1}+i f_{2}$ as in (1.4), where $f_{1}, f_{2} \in \mathbb{R}\left[x_{1}, y_{1}, \ldots, x_{d}, y_{d}\right]$. Let $F=f_{1}^{2}+f_{2}^{2}$. The zero set of $F$ in $\mathbb{S}^{d}$ is just $\mathrm{U}(f)$. The classical Łojasiewicz inequality applied to $F$ implies there are constants $C, \beta$ such that

$$
|\widehat{f}(\mathbf{s})|^{2}=|F(\mathbf{s})| \geqslant C \operatorname{dist}(\mathbf{s}, \mathrm{U}(f))^{\beta},
$$

where dist denotes the usual distance between points of $\mathbb{S}^{d}$. Since $\widehat{g}$ vanishes on $\mathrm{U}(f)$ and is Lipschitz, it follow that there is a $k \geqslant 1$ such that the function $G_{k}: \mathbb{S}^{d} \rightarrow \mathbb{C}$ defined by

$$
G_{k}(\mathbf{s})= \begin{cases}\frac{\widehat{g}(\mathbf{s})^{k}}{\widehat{f}(\mathbf{s})} & \text { if } \mathbf{s} \notin \mathrm{U}(f), \\ 0 & \text { if } \mathbf{s} \in \mathrm{U}(f)\end{cases}
$$

is continuous on $\mathbb{S}^{d}$. The standard formula for derivatives of quotients then shows that we can arrange for $G_{K}$ to have as many partial derivatives as we need by making $K$ large enough. Since sufficiently smooth functions have absolutely convergent Fourier series, $g^{K} \in \mathfrak{m}_{f}$ for large enough $K$, and so $g \in \sqrt{\mathfrak{m}_{f}}$.

We now state the main result of this section.

Theorem 3.2. Let $f \in R_{d}$ be a nonzero irreducible Laurent polynomial. Let $\alpha_{f}$ be the algebraic $\mathbb{Z}^{d}$-action on $X_{f}$ defined above, and $\Delta_{\alpha_{f}}^{1}\left(X_{f}\right)$ be the subgroup of summable homoclinic points of $\alpha_{f}$. Then the following are equivalent: 
(1) $\Delta_{\alpha_{f}}^{1}\left(X_{f}\right) \neq\{0\}$

(2) $\Delta_{\alpha_{f}}^{1}\left(X_{f}\right)$ is dense in $X_{f}$;

(3) $f$ is atoral (or, equivalently, $(f) \subsetneq \mathfrak{n}_{f}$ );

(4) $\operatorname{dim} \mathrm{U}(f) \leqslant d-2$.

Proof. The case $d=1$ is easily handled using the observations that $f \in R_{1}$ is atoral if and only if $\mathrm{U}(f)=\varnothing$, and this occurs if and only if $\alpha_{f}$ has nonzero homoclinic points which decay exponentially fast, in which case all homoclinic points are summable and the homoclinic group is dense (see [12] for details).

We may therefore assume that $d \geqslant 2$. The equivalence of (3) and (4) is contained in Proposition 2.2. Clearly (2) implies (1) since $f$ is not a unit and so $X_{f} \neq\{0\}$.

To prove the remaining implications, we first linearize the action $\alpha_{f}$. Consider the surjective map $\eta: \ell^{\infty}\left(\mathbb{Z}^{d}, \mathbb{R}\right) \rightarrow \mathbb{T}^{\mathbb{Z}^{d}}$ given by $\eta(w)_{\mathbf{n}}=w_{\mathbf{n}}(\bmod 1)$. We define the covering shift-action $\tilde{\sigma}$ of $\mathbb{Z}^{d}$ on $\ell^{\infty}\left(\mathbb{Z}^{d}, \mathbb{R}\right)$ by $\left(\tilde{\sigma}^{\mathbf{m}} w\right)_{\mathbf{n}}=w_{\mathbf{m}+\mathbf{n}}$. Set

$$
f(\tilde{\sigma})=\sum_{\mathbf{n}} f_{\mathbf{n}} \tilde{\sigma}^{\mathbf{n}}: \ell^{\infty}\left(\mathbb{Z}^{d}, \mathbb{R}\right) \rightarrow \ell^{\infty}\left(\mathbb{Z}^{d}, \mathbb{R}\right),
$$

and put

$$
\begin{aligned}
W_{f}:=\eta^{-1}\left(X_{f}\right) & =\left\{w \in \ell^{\infty}\left(\mathbb{Z}^{d}, \mathbb{R}\right): \eta(w) \in X_{f}\right\} \\
& =\left\{w \in \ell^{\infty}\left(\mathbb{Z}^{d}, \mathbb{R}\right): f(\tilde{\sigma})(w) \in \ell^{\infty}\left(\mathbb{Z}^{d}, \mathbb{Z}\right)\right\} .
\end{aligned}
$$

We view $W_{f}$ as the linearization of $X_{f}$. Also, viewing $f^{*}$ as an element of $\ell^{1}\left(\mathbb{Z}^{d}, \mathbb{R}\right)$, the point $f(\tilde{\sigma})(w)$ is the convolution product $f^{*} * w \in \ell^{\infty}\left(\mathbb{Z}^{d}, \mathbb{R}\right)$.

Now suppose (3) holds, so that $f$ is atoral. Hence there is a $g \in R_{d} \backslash(f)$ with $\mathrm{U}(g) \supseteq \mathrm{U}(f)=\mathrm{U}\left(f^{*}\right)$. By Lemma 3.1, there is a $k$ for which $\widehat{g}^{k} / \widehat{f}: \mathbb{S}^{d} \rightarrow \mathbb{C}$ has absolutely convergent Fourier transform $v=\left(v_{\mathbf{n}}\right) \in \ell^{1}\left(\mathbb{Z}^{d}, \mathbb{C}\right)$. Taking the Fourier transform of $\widehat{g}^{k} / \widehat{f}=\widehat{v}$ shows that $f * v=g^{k} \in \ell^{\infty}\left(\mathbb{Z}^{d}, \mathbb{Z}\right)$, and so $f^{*} * v^{*}=\left(g^{*}\right)^{k}$ has integral coordinates, so that $v^{*} \in W_{f}$. Hence the images under $\eta$ of the real and imaginary parts of $v^{*}$ give nonzero points in $\Delta_{\alpha_{f}}^{1}\left(X_{f}\right)$, proving (1).

Finally, suppose (1) holds, and let $0 \neq x \in \Delta_{\alpha_{f}}^{1}\left(X_{f}\right)$. Lift $x$ to $v \in W_{f} \cap \ell^{1}\left(\mathbb{Z}^{d}, \mathbb{R}\right)$ with $\eta(v)=x$. Since $v_{\mathbf{n}} \rightarrow 0$ as $\|\mathbf{n}\| \rightarrow \infty$, it follows that $f^{*} * v \in \ell^{\infty}\left(\mathbb{Z}^{d}, \mathbb{Z}\right)$ can have only finitely many nonzero coordinates, so represents a Laurent polynomial, say $h \in R_{d}$. Thus $\widehat{h}=\widehat{f^{*}} \cdot \widehat{v}$. This shows that $\widehat{h}$ vanishes on $\mathrm{U}\left(f^{*}\right)=\mathrm{U}(f)$, and so $h^{*} \in \mathfrak{n}_{f}$. If $h^{*}=g \cdot f$ for some $g \in R_{d}$, we would have $h=g^{*} \cdot f^{*}$, and so $\widehat{v} \widehat{f^{*}}=\widehat{g^{*}} \cdot \widehat{f^{*}}$, and a continuity argument shows that $v=g^{*} \in \ell^{\infty}\left(\mathbb{Z}^{d}, \mathbb{Z}\right)$. But then $x=\eta(v)=0$, a contradiction. Thus $h^{*} \in \mathfrak{n}_{f} \backslash(f)$, and so $f$ is atoral, proving (3). Furthermore $h$ is relatively prime to $f$, so that multiplication by $h$ is injective on $R_{d} /(f)$. It follows that $h\left(\alpha_{f}\right): X_{f} \rightarrow X_{f}$ is surjective. The proof of [13, Lemma 6.3] shows that the subgroup of $\Delta_{\alpha_{f}}^{1}\left(X_{f}\right)$ generated by the shifts of $x$ is dense in $h\left(\alpha_{f}\right)\left(X_{f}\right)=X_{f}$, proving (2) and completing the proof.

We extract one consequence of the preceding proof as a corollary. 
Corollary 3.3. Let $f \in R_{d}$ be an irreducible atoral polynomial, and let $\alpha_{f}$ be the algebraic $\mathbb{Z}^{d}$-action on $X_{f}$ as described above. Then every $x \in \Delta_{\alpha_{f}}^{1}\left(X_{f}\right)$ has the form $x_{h}=\eta\left(v_{h}\right)$, where $h^{*} \in \mathfrak{m}_{f}$ and $v_{h} \in \ell^{1}\left(\mathbb{Z}^{d}, \mathbb{R}\right)$ is the Fourier transform of $\widehat{h} / \widehat{f^{*}}$. Furthermore, $x_{h}$ is nonzero if and only if $h^{*} \in \mathfrak{m}_{f} \backslash(f)$, so that $\Delta_{\alpha_{f}}^{1}\left(X_{f}\right)$ is isomorphic as an abelian group to $\mathfrak{m}_{f} /(f)$.

In [12, Thm. 4.2] it is shown that, for expansive algebraic $\mathbb{Z}^{d}$-actions, the homoclinic group is dense if and only if the action has completely positive entropy. Using Theorem 3.2, these properties also hold for $\alpha_{f}$ when $f$ is atoral.

Corollary 3.4. Let $f \in R_{d}$ be a (possibly reducible) atoral Laurent polynomial that is not a unit in $R_{d}$. Then $\alpha_{f}$ has completely positive entropy and $\Delta_{\alpha_{f}}^{1}\left(X_{f}\right)$ is dense in $X_{f}$.

Proof. First recall from [4, Thm. 1] that an irreducible Laurent polynomial $g \in R_{d}$ has $\mathrm{h}\left(\alpha_{g}\right)=0$ if and only if there is a one-variable cyclotomic polynomial $c(t)$ and $\mathbf{m}, \mathbf{n} \in \mathbb{Z}^{d}$ such that $g(\mathbf{u})= \pm \mathbf{n}^{\mathbf{m}} c\left(\mathbf{u}^{\mathbf{n}}\right)$. For such $g$ clearly $\operatorname{dim} \mathbf{U}(g)=d-1$, and so $g$ is toral.

Let $f=f_{1}^{k_{1}} \cdots f_{r}^{k_{r}}$ be the factorization of $f$ into irreducible Laurent polynomials. The prime ideals associated with $(f)$ are just $\left(f_{1}\right), \ldots,\left(f_{r}\right)$, and $\mathrm{h}\left(\alpha_{R_{d} /\left(f_{j}\right)}\right)>0$ for $1 \leqslant j \leqslant r$ since each $f_{j}$ is atoral. Hence by [14, Thm. 6.5], it follows that $\alpha_{f}$ has completely positive entropy.

Suppose that $g$ and $h$ are relatively prime Laurent polynomials. Then

$$
X_{g h}=(g h)^{\perp}=[(g) \cap(h)]^{\perp}=(g)^{\perp}+(h)^{\perp}=X_{g}+X_{h} .
$$

Now by definition each $f_{j}$ is atoral, and so by the proof of Theorem 3.2 there is a $g_{j}$ for which $g_{j} / f_{j}$ has absolutely convergent Fourier series. Hence $g_{j}^{k_{j}} / f_{j}^{k_{j}}$ also has absolutely convergent Fourier series. If we denote $\alpha_{f_{j}^{k_{j}}}$ by $\alpha_{j}$ and $X_{f_{j}^{k_{j}}}$ by $X_{j}$, the proof of Theorem 3.2 shows that $\Delta_{\alpha_{j}}^{1}\left(X_{j}\right)$ is dense in $X_{j}$ for $1 \leqslant j \leqslant r$. Then $\Delta_{\alpha_{1}}^{1}\left(X_{1}\right)+\cdots+\Delta_{\alpha_{r}}^{1}\left(X_{r}\right)$ is contained in $\Delta_{\alpha_{f}}^{1}\left(X_{f}\right)$, and is dense in $X_{1}+\cdots+X_{r}$, which is equal to $X$ by (3.1).

\section{EXAmples}

We give here examples to illustrate various phenomena. For clarity, we use variables $u, v, w$ instead of $u_{1}, u_{2}, u_{3}$.

Example 4.1. Let $d=1$ and $f(u)=u^{2}-u-1$. Then $f(u)$ has roots $\lambda=(1+\sqrt{5}) / 2$ and $\mu=(1-\sqrt{5}) / 2$, so that $\mathrm{U}(f)=\varnothing$ and $f$ is atoral. As discussed in [12, Example 6.7 , we can compute the Fourier series of $1 / f^{*}(u)=1 /\left(u^{-1}-\lambda\right)\left(u^{-1}-\mu\right)$ by partial fractions and obtain the coefficients

$$
w_{n}^{\Delta}= \begin{cases}-\frac{1}{\sqrt{5}} \mu^{n-1} & \text { if } n \geqslant 1, \\ -\frac{1}{\sqrt{5}} \lambda^{n-1} & \text { if } n \leqslant 0 .\end{cases}
$$


Then $x^{\Delta}=\eta\left(w^{\Delta}\right) \in \Delta_{\alpha_{f}}^{1}\left(X_{f}\right)$, and $\Delta_{\alpha_{f}}\left(X_{f}\right)=\Delta_{\alpha_{f}}^{1}\left(X_{f}\right)$ is the group generated by all translates of $x^{\Delta}$.

Geometrically, $\alpha_{f}$ is a hyperbolic automorphism of the 2-dimensional torus $X_{f}$, and $\Delta_{\alpha_{f}}^{1}\left(X_{f}\right)$ is the dense intersection of the 1-dimensional stable and unstable eigenlines of $\alpha_{f}$.

Example 4.2. Let $d=1$ and $f(u)=u^{4}-u^{3}-u^{2}-u+1$. Then $\mathrm{U}(f)=\{\xi, \bar{\xi}\}$, where

$$
\xi=\frac{1-\sqrt{13}}{3}+\frac{1}{4} \sqrt{2+2 \sqrt{13}} i \in \mathbb{S}
$$

so that $f$ is toral. This can be seen directly since $f$ is the minimal polynomial of $\xi$ over $\mathbb{Q}$, so that any $g \in R_{1}$ with $\mathrm{U}(g) \supseteq \mathrm{U}(f)$ must be in $(f)$. As shown in [12, Example 3.4], $\Delta_{\alpha_{f}}\left(X_{f}\right)=\{0\}$, because the 1-dimensional stable and unstable eigenlines have only trivial intersection. Here $(f)=\mathfrak{n}_{f}=\mathfrak{m}_{f}$.

Example 4.3. Let $d=2$ and $f(u, v)=2-u-v$. Then $\mathrm{U}(f)=\{(1,1)\}$ and so $f$ is atoral. For example, $g(u, v)=u-1$ has $\mathrm{U}(g) \supseteq \mathrm{U}(f)$, but $g \notin(f)$.

As discussed in [13, Sect. 5], $1 / f^{*}$ is integrable on $\mathbb{S}^{2}$, with Fourier coefficients

$$
w_{(-m,-n)}^{\Delta}= \begin{cases}\frac{1}{2^{m+n+1}}\left(\begin{array}{c}
m+n \\
n
\end{array}\right) & \text { if } m \geqslant 0 \text { and } n \geqslant 0 \\
0 & \text { otherwise. }\end{cases}
$$

Hence $x^{\Delta}=\eta\left(w^{\Delta}\right) \in \Delta_{\alpha_{f}}\left(X_{f}\right)$, but $x^{\Delta}$ is not summable since, for example,

$$
x_{(-n,-n)}^{\Delta}=\frac{1}{2^{2 n+1}}\left(\begin{array}{c}
2 n \\
n
\end{array}\right) \approx \frac{1}{2 \sqrt{\pi n}}
$$

decays too slowly.

We can attempt to speed up the rate of decay by applying difference operators, or equivalently by multiplying $1 / f^{*}$ by powers of $u-1$ and $v-1$. It turns out that third powers are exactly what is needed, so that for example $(u-1)^{3} / f^{*}(u, v)$ has absolutely convergent Fourier series whose coefficients provide a nonzero point in $\Delta_{\alpha_{f}}^{1}\left(X_{f}\right)$.

Here $\mathfrak{n}_{f}=\left\{g \in R_{2}: g(1,1)=\sum_{\mathbf{n}} g_{\mathbf{n}}=0\right\}$ and $\mathfrak{m}_{f}$ is the ideal in $R_{2}$ generated by $(u-1)^{p}(v-1)^{q}$ with $p+q=3$. The "summable defect" in this example is

$$
\Delta_{\alpha_{f}}\left(X_{f}\right) / \Delta_{\alpha_{f}}^{1}\left(X_{f}\right) \cong \mathfrak{n}_{f} / \mathfrak{m}_{f},
$$

which is a finitely generated abelian group of rank five generated by the cosets $(u-1)^{r}(v-1)^{s}+\mathfrak{m}_{f}$ with $1 \leqslant r+s \leqslant 2$.

Example 4.4. Let $d=2$ and $f(u, v)=3-u-u^{-1}-v-v^{-1}$. Then $\mathrm{U}(f)$ is a 1-dimensional curve in $\mathbb{S}^{2}$ which is the image under the exponential map $e: \mathbb{T}^{2} \rightarrow \mathbb{S}^{2}$ of the closed curve given by

$$
t= \pm \frac{1}{2 \pi} \cos ^{-1}\left(\frac{3}{2}-\cos 2 \pi s\right), \quad-\frac{1}{6} \leqslant s \leqslant \frac{1}{6} .
$$

Thus $f$ is toral, and so $\Delta_{\alpha_{f}}^{1}\left(X_{f}\right)=\{0\}$. It follows easily from [15] that all elements of $\mathrm{U}(f) \cap \Omega$ must have order 30. A straightforward search verifies that $\mathrm{U}(f) \cap$ 
$\Omega=\{(\omega, 1),(\bar{\omega}, 1),(1, \omega),(1, \bar{\omega})\}$, where $\omega=e^{2 \pi i / 6}$. Hence $\operatorname{dim} \operatorname{Fix}_{\Gamma}^{\circ}\left(\alpha_{f}\right) \leqslant 4$ for all $\Gamma \in \mathcal{F}$.

Ironically, $\Delta_{\alpha_{f}}\left(X_{f}\right)$ is so large that it is uncountable. Let $\mu$ be any measure supported on $\mathrm{U}(f)$ that is a smooth function multiple of arc length. Since the curvature of $\mathrm{U}(f)$ is bounded away from zero, a result of Stein [21, Thm. 2 of $\S$ VIII.3.2] shows that $\widehat{\mu}(\mathbf{n}) \rightarrow 0$ as $\|\mathbf{n}\| \rightarrow \infty$. Hence the point $x$ with $x_{\mathbf{n}}=$ $\eta(\operatorname{Re}[\widehat{\mu}(\mathbf{n})])$ is in $\Delta_{\alpha_{f}}\left(X_{f}\right)$. However, the same result shows that $\widehat{\mu}(\mathbf{n})$ must decay so slowly that $x$ is never summable.

The dynamical properties of $\alpha_{f}$ are still somewhat mysterious. In particular, we do not know whether Theorem 1.3 holds here.

Example 4.5. Let $d=2$ and $f(u, v)=u^{2}+u^{-2}-2 u-2 u^{-1}+v+v^{-1}$. Then $f$ is irreducible, and it is easy to check that $\mathrm{U}(f)$ consists of two 1-dimensional curves together with the point $(1,1)$. This illustrates the possibility that the connected components of $\mathrm{U}(f)$ may have different dimensions.

Example 4.6. Let $d=3$ and $f(u, v, w)=1+u+v+w$. This example has appeared in the literature due to the interesting value of its logarithmic Mahler measure [20]: $\mathrm{m}(f)=7 \zeta(3) / 2 \pi^{2}$. It is easy to verify that $\mathrm{U}(f)$ is the union of three circles $\{(-1, s,-s): s \in \mathbb{S}\},\{(s,-1,-s): s \in \mathbb{S}\}$, and $\{(s,-s,-1): s \in \mathbb{S}\}$. Hence $f$ is atoral. For example, both $u v w+u v+u w+v w$ and $(u-v)(v-w)(w-u)$ vanish on $\mathrm{U}(f)$ but are not in $(f)$.

Observe that $\mathrm{U}(f)$ contains infinitely many torsion points. Indeed, if $\Gamma_{n}=n \mathbb{Z}^{3}$, then

$$
\left|\Omega_{\Gamma_{n}} \cap \mathrm{U}(f)\right|= \begin{cases}3 n-3 & \text { if } n \text { is even } \\ 0 & \text { if } n \text { is odd. }\end{cases}
$$

Hence the dimension of $\mathrm{Fix}_{\Gamma_{n}}^{\circ}\left(\alpha_{f}\right)$ is unbounded, an issue that will need to be dealt with in the proof of our main theorem (see Lemma 6.8).

This example is a special case of the fact that if $\mathrm{U}(f)$ has infinitely many torsion points, then all of these must lie on a finite union of cosets of rational subtori.

Example 4.7. Let $d=3$ and $f(u, v, w)=2+u+v+w$, which is irreducible. Since $f$ is not symmetric, it is atoral. One can consider points in $\mathrm{U}(f)$ as possible positions of a closed linkage of four rods, one fixed horizontal rod of length 2 , and three others of length 1 joined end to end. This system has one degree of freedom, and so $\mathrm{U}(f)$ is a smooth loop in $\mathbb{S}^{3}$.

By Theorem 3.2, $\Delta_{\alpha_{f}}^{1}\left(X_{f}\right) \neq\{0\}$ (in fact is dense in $X_{f}$ ). Now $\mathrm{U}(f)$ is a smooth curve with finite order of contact with every hyperplane (i.e., has finite type in the terminology of [21]). Hence if $\mu$ is any measure on $\mathrm{U}(f)$ that is a smooth multiple of arc length, then by [21, Thm. 2, §VIII.3.2] we have that $\widehat{\mu}(\mathbf{n}) \rightarrow 0$ as $\|\mathbf{n}\| \rightarrow \infty$. Hence $x=\left(x_{\mathbf{n}}\right)$, where $x_{\mathbf{n}}=\eta(\operatorname{Re}[\widehat{\mu}(\mathbf{n})])$, is in $\Delta_{\alpha_{f}}\left(X_{f}\right)$, but decays too slowly to be summable.

In this example $\Delta_{\alpha_{f}}^{1}\left(X_{f}\right)$ is countable and dense in $X_{f}$ by Corollary 3.4, while $\Delta_{\alpha_{f}}\left(X_{f}\right) \backslash \Delta_{\alpha_{f}}^{1}\left(X_{f}\right)$ is uncountable. 
Example 4.8. The roots of $u^{2}-9 u+19$ are $\xi \approx 3.3819$ and $\zeta \approx 5.6180$. Consider

$$
\begin{gathered}
f(u, v):=g_{1}(u, v) g_{2}(u, v)=\left(\xi-u-u^{-1}-v-v^{-1}\right)\left(\zeta-u-u^{-1}-v-v^{-1}\right) \\
=23+u^{2}+u^{-2}-9 u-9 u^{-1}+v^{2}+v^{-2}-9 v-9 v^{-1} \\
+2 u v+2 u^{-1} v^{-1}+2 u v^{-1}+2 u^{-1} v .
\end{gathered}
$$

Here $f$ is irreducible in $R_{2}$, or equivalently in $\mathbb{Q}\left[u^{ \pm 1}, v^{ \pm 1}\right]$, but factors in $\mathbb{C}\left[u^{ \pm 1}, v^{ \pm 1}\right]$. Furthermore, $\mathrm{U}\left(g_{1}\right)$ is a 1-dimensional curve in $\mathbb{S}^{2}$, analogous to Example 4.4, while $\mathrm{U}\left(g_{2}\right)=\varnothing$, and so $f$ is toral in our sense. However, $f$ is neither toral nor atoral in the sense of [1] since it has mixed factors over $\mathbb{C}$.

Remark 4.9. If $f \in R_{d}$ is irreducible, but factors over $\mathbb{C}$, it can be shown that except for a trivial scalar normalization, each factor has coefficients that are algebraic numbers, i.e. this factorization already takes place in $\overline{\mathbb{Q}}\left[u_{1}^{ \pm 1}, \ldots, u_{d}^{ \pm 1}\right]$. Indeed, by Dedekind's Prague Theorem [8, p. 2], the coefficients of each factor can be taken to be algebraic integers.

\section{Symbolic COVERS AND SPECIFICATION}

Let $f \in R_{d}$ be an irreducible atoral Laurent polynomial and $\alpha_{f}$ be the corresponding algebraic $\mathbb{Z}^{d}$-action on $X_{f}$. By Theorem 3.2, there are nonzero summable homoclinic points for $\alpha_{f}$. Fix one of these, say $x$. As pointed out in [12], there is a surjective, shift-equivariant map $\xi_{x}: \ell^{\infty}\left(\mathbb{Z}^{d}, \mathbb{Z}\right) \rightarrow X_{f}$ defined by

$$
\xi_{x}(v)=\sum_{\mathbf{n} \in \mathbb{Z}^{d}} v_{\mathbf{n}} \alpha_{f}^{-\mathbf{n}}(x),
$$

where coordinatewise convergence follows from summability of $x$. In fact, $\xi_{x}$ is surjective when restricted to a suitably large ball of radius $K$ in $\ell^{\infty}\left(\mathbb{Z}^{d}, \mathbb{Z}\right)$, thereby providing a symbolic cover for $X_{f}$ with symbols $\{-K, \ldots, K\}$.

Having established the existence of summable homoclinic points for atoral polynomials here, the proof in [13, Thm. 8.2] using symbolic covers applies to yield the following remarkably strong specification properties of $\alpha_{f}$.

Proposition 5.1 ([13, Thm. 8.2]). Let $f \in R_{d}$ be an irreducible atoral polynomial and $\alpha_{f}$ be the corresponding algebraic $\mathbb{Z}^{d}$-action on $X_{f}$. Fix a translation-invariant metric $\delta$ on $X_{f}$. Then for every $\varepsilon>0$ there exists a number $p(\varepsilon)>0$ with the following properties:

(1) For every finite collection $\left\{Q_{1}, \ldots, Q_{r}\right\}$ of finite subsets of $\mathbb{Z}^{d}$ with

$\left(^{*}\right) \quad \operatorname{dist}\left(Q_{j}, Q_{k}\right):=\min _{\mathbf{m} \in Q_{j}, \mathbf{n} \in Q_{k}}\|\mathbf{m}-\mathbf{n}\| \geqslant p(\varepsilon)$ for $1 \leqslant j<k \leqslant r$,

every collection $\left\{x^{(1)}, \ldots, x^{(r)}\right\} \subset X_{f}$, and every $\Gamma \in \mathcal{F}$ with

$$
\operatorname{dist}\left(Q_{j}+\mathbf{k}, Q_{k}\right) \geqslant p(\varepsilon) \text { for } 1 \leqslant j<k \leqslant r \text { and every } \mathbf{k} \in \Gamma \backslash\{\mathbf{0}\} \text {, }
$$

there is a $y \in \operatorname{Fix}_{\Gamma}\left(\alpha_{f}\right)$ with

$$
\delta\left(\alpha_{f}^{\mathbf{n}}(y), \alpha_{f}^{\mathbf{n}}\left(x^{(j)}\right)\right)<\varepsilon \text { for } 1 \leqslant j \leqslant r \text { and every } \mathbf{n} \in Q_{j} .
$$


(2) For every finite collection $\left\{Q_{1}, \ldots, Q_{r}\right\}$ of finite subsets of $\mathbb{Z}^{d}$ satisfying $(*)$ and every collection $\left\{x^{(1)}, \ldots, x^{(r)}\right\} \subset X_{f}$ there is a point $y \in \Delta_{\alpha_{f}}^{1}\left(X_{f}\right)$ satisfying $(* *)$.

\section{Proof of the MAin theorem}

To begin the proof of Theorem 1.3, we fix an atoral irreducible Laurent polynomial $f \in R_{d}$, and let $\alpha_{f}$ be the corresponding cyclic algebraic $\mathbb{Z}^{d}$-action on $X_{f}$.

Roughly speaking, with the availability of summable homoclinic points, obtaining sufficiently many separated points in $\operatorname{Fix}_{\Gamma}\left(\alpha_{f}\right)$ is relatively easy. However, many of these could lie in the same coset of the connected component Fix ${ }_{\Gamma}^{\circ}\left(\alpha_{f}\right)$ of the identity. In order to show that this does not affect the logarithmic growth rate of $\mathrm{P}_{\Gamma}\left(\alpha_{f}\right)$, we invoke a result of $\mathrm{H}$. B. Mann which shows that $\mathrm{U}(f) \cap \Omega$ lies in the union of a finite number of cosets of rational subtori of $\mathbb{S}^{d}$. This enables us to embed $\operatorname{Fix}_{\Gamma}^{\circ}\left(\alpha_{f}\right)$ in a finite sum of subtori with spanning sets whose cardinality have logarithmic growth rate zero. This will force the number of cosets of $\operatorname{Fix}_{\Gamma}^{\circ}\left(\alpha_{f}\right)$ in $\operatorname{Fix}_{\Gamma}\left(\alpha_{f}\right)$ to have the correct logarithmic growth rate.

We begin with some terminology.

Definition 6.1. Let $Q \subset \mathbb{Z}^{d}$ be a finite set. Define a pseudometric $\mathrm{d}_{Q}$ on $\mathbb{T}^{\mathbb{Z}^{d}}$ by setting

$$
\mathrm{d}_{Q}(x, y)=\max _{\mathbf{n} \in Q}\left\|x_{\mathbf{n}}-y_{\mathbf{n}}\right\|, \quad \text { for } x, y \in \mathbb{T}^{\mathbb{Z}^{d}},
$$

where $\|x-y\|$ is the usual distance between $s, t \in \mathbb{T}$.

A set $F \subset \mathbb{T}^{\mathbb{Z}^{d}}$ is $(Q, \varepsilon)$-separated if $\mathrm{d}_{Q}(x, y)>\varepsilon$ for every pair $x, y$ of distinct points in $F$. If $Y \subset \mathbb{T}^{\mathbb{Z}^{d}}$, then a set $F \subset Y$ is $(Q, \varepsilon)$-spanning if, for every $y \in Y$, there is an $x \in F$ with $\mathrm{d}_{Q}(x, y)<\varepsilon$.

If $\Gamma \in \mathcal{F}$, we write $\mathrm{d}_{\Gamma}$ for the metric on $\operatorname{Fix}_{\Gamma}(\sigma)$ defined by $\mathrm{d}_{\Gamma}=\mathrm{d}_{Q}$ for any fundamental domain $Q$ for $\Gamma$. A set $F \subset \operatorname{Fix}_{\Gamma}(\sigma)$ is $(\Gamma, \varepsilon)$-separated if $\mathrm{d}_{\Gamma}(x, y)>\varepsilon$ for every pair $x, y$ of distinct points in $F$, and there is an analogous definition of $(\Gamma, \varepsilon)$-spanning.

Lemma 6.2. For every $\varepsilon>0$ there is a finite set $A_{\varepsilon} \subset \mathbb{Z}^{d}$ such that, for every $\Gamma \in \mathcal{F}$ and every fundamental domain $Q \subset \mathbb{Z}^{d}$ of $\Gamma$, the set $\operatorname{Fix}_{\Gamma}\left(\alpha_{f}\right)$ is $\left(\bigcap_{\mathbf{m} \in A_{\varepsilon}}(Q-\mathbf{m})\right)$ spanning in $X_{f}$.

Proof. Since $f$ is atoral, our discussion in Section 3 shows that there is a $g \in R_{d}$ relatively prime to $f$ such that the Fourier transform of $g / f$ provides a summable homoclinic point for $\alpha_{f}$. The proof of Lemma 7.3 in [13] is then also valid in this situation.

The next lemma is an easily proved special case of [6, Cor. 5.6].

Lemma 6.3. Let $\left(\Gamma_{n}\right)_{n \geqslant 1}$ be a sequence in $\mathcal{F}$ with $\left\langle\Gamma_{n}\right\rangle \rightarrow \infty$ as $n \rightarrow \infty$. Then there exists a sequence $\left(Q_{n}\right)_{n \geqslant 1}$ of finite subsets of $\mathbb{Z}^{d}$ such that each $Q_{n}$ is a fundamental domain for $\Gamma_{n}$, and also that $\left(Q_{n}\right)_{n \geqslant 1}$ is a Følner sequence for $\mathbb{Z}^{d}$. 
Lemma 6.4. Let $\varepsilon>0$ and let $\left(\Gamma_{n}\right)_{n \geqslant 1}$ be a sequence in $\mathcal{F}$ with $\left\langle\Gamma_{n}\right\rangle \rightarrow \infty$. Choose a Følner sequence $\left(Q_{n}\right)_{n \geqslant 1}$ of fundamental domains for the groups $\left(\Gamma_{n}\right)_{n \geqslant 1}$ as in Lemma 6.3. Let $A_{\varepsilon}$ be the finite set chosen according to Lemma 6.2. Define

$$
Q_{n}^{\prime}=\bigcap_{\mathbf{m} \in A_{\varepsilon}}\left(Q_{n}-\mathbf{m}\right) \text {. }
$$

Then $\left(Q_{n}^{\prime}\right)_{n \geqslant 1}$ is again a Følner sequence, and $\left|Q_{n}^{\prime}\right| /\left|Q_{n}\right| \rightarrow 1$ as $n \rightarrow \infty$. Furthermore, for every $n \geqslant 1$ there exists a $\left(\Gamma_{n}, \varepsilon\right)$-separated set $F_{n}(\varepsilon) \subset \operatorname{Fix}_{\Gamma_{n}}\left(\alpha_{f}\right)$ which is $\left(Q_{n}^{\prime}, 2 \varepsilon\right)$-spanning in $X_{f}$.

Proof. From Lemma 6.2 we know that $\operatorname{Fix}_{\Gamma_{n}}\left(\alpha_{f}\right)$ is $\left(Q_{n}^{\prime}, \varepsilon\right)$-spanning in $X_{f}$ for all $n \geqslant 1$. Let $F_{n}(\varepsilon) \subset \operatorname{Fix}_{\Gamma_{n}}\left(\alpha_{f}\right)$ be a $(\Gamma, \varepsilon)$-separated set of maximal cardinality. Then $F_{n}(\varepsilon)$ is also $\left(\Gamma_{n}, \varepsilon\right)$-spanning in $\operatorname{Fix}_{\Gamma_{n}}\left(\alpha_{f}\right)$, hence $\left(Q_{n}^{\prime}, 2 \varepsilon\right)$-spanning in $X_{f}$.

It follows from [5, Prop. 2] that

$$
\lim _{\varepsilon \rightarrow 0} \liminf _{n \rightarrow \infty} \frac{1}{\left|\mathbb{Z}^{d} / \Gamma_{n}\right|} \log \left|F_{n}(\varepsilon)\right|=\mathrm{h}\left(\alpha_{f}\right) .
$$

The next, and more difficult, step in our proof consists of showing that the number of distinct cosets of $\mathrm{Fix}_{\Gamma_{n}}^{\circ}\left(\alpha_{f}\right)$ intersecting $F_{n}(\varepsilon)$ nontrivially has the same logarithmic growth rate as $F_{n}(\varepsilon)$.

We first introduce some notation to linearize our situation. We write $\ell^{\infty}\left(\mathbb{Z}^{d}, \mathbb{C}\right)$ for the space of bounded complex-valued functions on $\mathbb{Z}^{d}$, and $\tilde{\sigma}$ for the $\mathbb{Z}^{d}$-shift action on this space. Similarly, $\tilde{\sigma}$ acts on the real part $\ell^{\infty}\left(\mathbb{Z}^{d}, \mathbb{R}\right)$ of $\ell^{\infty}\left(\mathbb{Z}^{d}, \mathbb{C}\right)$. For $\Gamma \in \mathcal{F}$ we let $\ell\left(\mathbb{Z}^{d} / \Gamma, \mathbb{C}\right)$ and $\ell\left(\mathbb{Z}^{d} / \Gamma, \mathbb{R}\right)$ be the corresponding finite-dimensional $\Gamma$ periodic subspaces. For each $\boldsymbol{\omega} \in \Omega_{\Gamma}$ there is an element $v^{(\boldsymbol{\omega})}$ in $\ell\left(\mathbb{Z}^{d} / \Gamma, \mathbb{C}\right)$ defined by $v_{\mathbf{n}}^{(\boldsymbol{\omega})}=\boldsymbol{\omega}^{\mathbf{n}}$ for all $\mathbf{n} \in \mathbb{Z}^{d}$.

A set $S \subset \Omega_{\Gamma}$ is called symmetric if it is closed under taking inverses. A function $c: S \rightarrow \mathbb{C}$ on a symmetric set is skew-symmetric if $c\left(\boldsymbol{\omega}^{-1}\right)=\overline{c(\boldsymbol{\omega})}$. Let $V(S, \mathbb{C}) \subset$ $\ell\left(\mathbb{Z}^{d} / \Gamma, \mathbb{C}\right)$ denote the complex span of the points $v^{(\boldsymbol{\omega})}$ where $\boldsymbol{\omega} \in S$, and $V(S, \mathbb{R})=$ $V(S, \mathbb{C}) \cap \ell\left(\mathbb{Z}^{d} / \Gamma, \mathbb{R}\right)$. Then $V(S, \mathbb{R})$ consists of all sums of the form $\sum_{\boldsymbol{\omega} \in S} c(\boldsymbol{\omega}) v^{(\boldsymbol{\omega})}$ where $c$ is skew-symmetric on $S$, which has real dimension $|S|$.

For $\Delta \in \mathcal{F}$ let $B_{1}\left(\ell\left(\mathbb{Z}^{d} / \Delta, \mathbb{R}\right)\right)$ denote the unit ball in $\ell\left(\mathbb{Z}^{d} / \Delta, \mathbb{R}\right)$ with respect to the $\ell^{\infty}$-norm.

Lemma 6.5. Let $\Delta \in \mathcal{F}$ and $0<\varepsilon<1$. Then there is a $(\Delta, \varepsilon)$-spanning set $F \subset B_{1}\left(\ell\left(\mathbb{Z}^{d} / \Delta, \mathbb{R}\right)\right)$ with cardinality $|F|<(2 / \varepsilon)^{\left|\mathbb{Z}^{d} / \Delta\right|}$. Hence $\eta\left(\ell\left(\mathbb{Z}^{d} / \Delta, \mathbb{R}\right)\right)=$ $\operatorname{Fix}_{\Delta}(\sigma)=\left\{x \in \mathbb{T}^{\mathbb{Z}^{d}}: \sigma^{\mathbf{n}} x=x\right.$ for all $\left.\mathbf{n} \in \Delta\right\}$ has a $(\Delta, \varepsilon)$-spanning set of cardinality $<(2 / \varepsilon)^{\left|\mathbb{Z}^{d} / \Delta\right|}$.

Proof. Let $Q$ be a fundamental domain for $\Delta$. Then $\ell\left(\mathbb{Z}^{d} / \Delta, \mathbb{R}\right) \cong \ell^{\infty}(Q, \mathbb{R})$ with the $\ell^{\infty}$-norm. For each $\mathbf{q} \in Q$ let $F_{\mathbf{q}}=\left\{j \varepsilon 1_{\{\mathbf{q}\}}:-1 / \varepsilon<j<1 / \varepsilon\right\}$, so $\left|F_{\mathbf{q}}\right|<2 / \varepsilon$. Put $F=\sum_{\mathbf{q} \in Q} F_{\mathbf{q}}$. Clearly $F$ is $(\Delta, \varepsilon)$-spanning for $B_{1}\left(\ell\left(\mathbb{Z}^{d} / \Delta, \mathbb{R}\right)\right)$, and $|F|<$ $(2 / \varepsilon)^{|Q|}=(2 / \varepsilon)^{\left|\mathbb{Z}^{d} / \Delta\right|}$.

Finally, $\eta\left(B_{1}\left(\ell\left(\mathbb{Z}^{d} / \Delta, \mathbb{R}\right)\right)\right)=\mathrm{Fix}_{\Delta}(\sigma)$ and $\eta$ is a local isometry. Hence $\eta(F)$ is a $(\Delta, \varepsilon)$-spanning set for $\operatorname{Fix}_{\Delta}(\sigma)$ of cardinality $<(2 / \varepsilon)^{\left|\mathbb{Z}^{d} / \Delta\right|}$. 
We will be using proper closed subgroups of $\mathbb{S}^{d}$ to capture the torsion points in $\mathrm{U}(f)$, which are responsible for the dimension of $\operatorname{Fix}_{\Gamma}^{\circ}\left(\alpha_{f}\right)$. To do so, we define, for every $\mathbf{0} \neq \mathbf{m} \in \mathbb{Z}^{d}$ the subgroup $H_{\mathbf{m}}=\left\{\mathbf{s} \in \mathbb{S}^{d}: \mathbf{s}^{\mathbf{m}}=1\right\} \subset \mathbb{S}^{d}$. Observe that $H_{\mathbf{m}}^{\perp}:=\left\{\mathbf{n} \in \mathbb{Z}^{d}: \mathbf{s}^{\mathbf{n}}=1\right.$ for all $\left.\mathbf{s} \in H_{\mathbf{m}}\right\}$ is just $\mathbb{Z} \mathbf{m}$. The next lemma allows us to estimate the size of the slice of $\Omega_{\Gamma}$ contained in $H_{\mathbf{m}}$.

Lemma 6.6. Let $\mathbf{0} \neq \mathbf{m} \in \mathbb{Z}^{d}$ and $\Gamma \in \mathcal{F}$. Then

$$
\left|\Omega_{\Gamma} \cap H_{\mathbf{m}}\right| \leqslant \frac{\|\mathbf{m}\|}{\langle\Gamma\rangle}\left|\Omega_{\Gamma}\right|
$$

Proof. Basic duality shows that $\Omega_{\Gamma} /\left(\Omega_{\Gamma} \cap H_{\mathbf{m}}\right)$ is isomorphic with $\left(\Omega_{\Gamma} \cap H_{\mathbf{m}}\right)^{\perp} / \Omega_{\Gamma}^{\perp}$, and that $\Omega_{\Gamma}^{\perp}=\Gamma$ and $\left(\Omega_{\Gamma} \cap H_{\mathbf{m}}\right)^{\perp}=\Omega_{\Gamma}^{\perp}+H_{\mathbf{m}}^{\perp}=\Gamma+\mathbb{Z} \mathbf{m}$. Hence $\left|\Omega_{\Gamma} /\left(\Omega_{\Gamma} \cap H_{\mathbf{m}}\right)\right|=$ $|(\Gamma+\mathbb{Z} \mathbf{m}) / \Gamma|$, which is just the order $k$ of $\mathbf{m}$ in $\mathbb{Z}^{d} / \Gamma$. Since $\mathbf{0} \neq k \mathbf{m} \in \Gamma$, we have $\|k \mathbf{m}\| \geqslant\langle\Gamma\rangle$, so that $k \geqslant\langle\Gamma\rangle /\|\mathbf{m}\|$. Hence

$$
\left|\Omega_{\Gamma} \cap H_{\mathbf{m}}\right|=\frac{1}{k}\left|\Omega_{\Gamma}\right| \leqslant \frac{\langle\Gamma\rangle}{\|\mathbf{m}\|}\left|\Omega_{\Gamma}\right| .
$$

Lemma 6.7. Let $\mathbf{0} \neq \mathbf{m} \in \mathbb{Z}^{d}$ and $\mathbf{s} \in \mathbb{S}^{d}$. If $\left(\mathbf{s} \cdot H_{\mathbf{m}}\right) \cap \Omega \neq \varnothing$, then there is a $k \geqslant 1$ such that $\mathbf{s} \cdot H_{\mathbf{m}} \subset H_{k \mathbf{m}}$.

Proof. If $\mathbf{s} \cdot H_{\mathbf{m}} \cap \Omega \neq \varnothing$, then there is an $\mathbf{s}^{\prime} \in \Omega$ such that $\mathbf{s}^{\prime} \cdot H_{\mathbf{m}}=\mathbf{s} \cdot H_{\mathbf{m}}$. Let $k$ be the order of $\mathbf{s}^{\prime}$ in $\mathbb{S}^{d}$.

Lemma 6.8. Let $0 \neq f \in R_{d}$. Then there are nonzero $\mathbf{m}_{1}, \ldots, \mathbf{m}_{L} \in \mathbb{Z}^{d}$ such that

$$
\mathrm{U}(f) \cap \Omega \subset \bigcup_{j=1}^{L} H_{\mathbf{m}_{j}} .
$$

Proof. A special case of the Manin-Mumford conjecture established by Mann [15] shows that $\mathrm{U}(f) \cap \Omega$ is contained in a finite union of cosets of connected proper rational subtori (i.e., closed, connected subgroups) of $\mathbb{S}^{d}$. By Lemma 6.7, we can embed these cosets into appropriate $H_{\mathbf{m}_{j}}$.

Remark 6.9. There is a finite procedure for computing the $\mathbf{m}_{j}$ appearing in the previous lemma (see [10, §3.1] or [2, §6]).

Lemma 6.10. Let $\Gamma \in \mathcal{F}$ and $0 \neq f \in R_{d}$. Define $f(\tilde{\sigma}): \ell^{\infty}\left(\mathbb{Z}^{d}, \mathbb{R}\right) \rightarrow \ell^{\infty}\left(\mathbb{Z}^{d}, \mathbb{R}\right)$ as above, and put $V_{\Gamma}(f)=\operatorname{ker} f(\tilde{\sigma}) \cap \ell\left(\mathbb{Z}^{d} / \Gamma, \mathbb{R}\right)$. Then $V_{\Gamma}(f)=V\left(\mathrm{U}(f) \cap \Omega_{\Gamma}, \mathbb{R}\right)$ and $\eta\left(V_{\Gamma}(f)\right)=\operatorname{Fix}_{\Gamma}^{\circ}\left(\alpha_{f}\right)$.

Proof. This assertion is contained in the proof of [13, Lemma 6.8], but we include it here for the convenience of the reader. Since $f$ has real coefficients, $\mathrm{U}(f) \cap$ $\Omega_{\Gamma}$ is symmetric. Hence $V:=V\left(\mathrm{U}(f) \cap \Omega_{\Gamma}, \mathbb{R}\right)$ consists of the skew-symmetric combinations $\sum_{\boldsymbol{\omega} \in \mathrm{U}(f) \cap \Omega_{\Gamma}} c(\boldsymbol{\omega}) v^{(\boldsymbol{\omega})}$, so the first statement follows. Clearly $V$ is a linear subspace of $\ell^{\infty}\left(\mathbb{Z}^{d}, \mathbb{R}\right)$ and $\eta(V) \subset \operatorname{Fix}_{\Gamma}^{\circ}\left(\alpha_{f}\right)$ by connectedness.

Let $\varepsilon=\left(2 \sum_{\mathbf{n} \in \mathbb{Z}^{d}}\left|f_{\mathbf{n}}\right|\right)^{-1}$, and consider the neighborhood $N_{\varepsilon}=\left\{x \in \operatorname{Fix}_{\Gamma}^{\circ}\left(\alpha_{f}\right)\right.$ : $\left.\sup _{\mathbf{n}}\left\|x_{\mathbf{n}}\right\|<\varepsilon\right\}$. If $\widetilde{B}_{\varepsilon}=\left\{v \in \ell\left(\mathbb{Z}^{d} / \Gamma, \mathbb{R}\right):\|v\|_{\infty}<\varepsilon\right\}$ and $\widetilde{N}_{\varepsilon}=\eta^{-1}\left(X_{f}\right) \cap \widetilde{B}_{\varepsilon}$, 
then $\eta\left(\widetilde{N}_{\varepsilon}\right)=N_{\varepsilon}$. For every $v \in \widetilde{N}_{\varepsilon}$ we have that $\eta(f(\tilde{\sigma}) v)=0$, so that $f(\tilde{\sigma})(v) \in$ $\ell\left(\mathbb{Z}^{d} / \Gamma, \mathbb{Z}\right)$. But our choice of $\varepsilon$ then forces $f(\tilde{\sigma})(v)=0$, so that $v \in V$. Hence $\eta(V) \supset N_{\varepsilon}$. Since $\operatorname{Fix}_{\Gamma}^{\circ}\left(\alpha_{f}\right)$ is connected, it follows that $\eta(V) \supset \operatorname{Fix}_{\Gamma}^{\circ}\left(\alpha_{f}\right)$, and hence $\eta(V)=\operatorname{Fix}_{\Gamma}^{\circ}\left(\alpha_{f}\right)$.

Lemma 6.11. Let $0 \neq f \in R_{d}$ and choose nonzero $\mathbf{m}_{1}, \ldots, \mathbf{m}_{L} \in \mathbb{Z}^{d}$ according to Lemma 6.8 so that $\mathrm{U}(f) \cap \Omega \subset H_{\mathbf{m}_{1}} \cup \cdots \cup H_{\mathbf{m}_{L}}$. Let $\Delta_{\Gamma, j}=\left(\Omega_{\Gamma} \cap H_{\mathbf{m}_{j}}\right)^{\perp} \supset \Gamma$. Then

$$
\operatorname{Fix}_{\Gamma}^{\circ}\left(\alpha_{f}\right) \subset \sum_{j=1}^{L} \operatorname{Fix}_{\Delta_{\Gamma, j}}(\sigma) .
$$

Proof. Let $S_{j}=\Omega_{\Gamma} \cap H_{\mathbf{m}_{j}}$ and $S=S_{1} \cup \ldots S_{L}$, which is clearly symmetric. Then

$$
\begin{aligned}
\operatorname{Fix}_{\Gamma}^{\circ}\left(\alpha_{f}\right) & =\eta\left(V\left(\Omega_{\Gamma} \cap \mathrm{U}(f), \mathbb{R}\right) \subset \eta(V(S, \mathbb{R}))=\sum_{j=1}^{L} \eta\left(V\left(S_{j}, \mathbb{R}\right)\right)\right. \\
& =\sum_{j=1}^{L} \eta\left(\ell\left(\mathbb{Z}^{d} / \Delta_{\Gamma, j}, \mathbb{R}\right)\right)=\sum_{j=1}^{L} \operatorname{Fix}_{\Delta_{\Gamma, j}}(\sigma) .
\end{aligned}
$$

Proof of Theorem 1.3. Let $\left(\Gamma_{n}\right)_{n \geqslant 1}$ be a sequence in $\mathcal{F}$ with $\left\langle\Gamma_{n}\right\rangle \rightarrow \infty$, and fix $\varepsilon>0$ for the moment. Chose $\mathbf{m}_{1}, \ldots, \mathbf{m}_{L} \in \mathbb{Z}^{d}$ for $f$ according to Lemma 6.8. Put $M=\max _{1 \leqslant j \leqslant L}\left\|\mathbf{m}_{j}\right\|$. Let $\Delta_{n, j}=\left(\Omega_{\Gamma_{n}} \cap H_{\mathbf{m}_{j}}\right)^{\perp} \supset \Gamma_{n}$.

Applying Lemma 6.5 to each $\Delta_{n, j}$, we obtain $\left(\Delta_{n, j}, \varepsilon / 2 L\right)$-spanning (and hence $\left(\Gamma_{n}, \varepsilon / 2 L\right)$-spanning) sets $F_{n, j} \subset \mathrm{Fix}_{\Delta_{n, j}}(\sigma)$ of cardinality $\left|F_{n, j}\right|<(4 L / \varepsilon)^{\left|\mathbb{Z}^{d} / \Delta_{n, j}\right|}$. Let $F_{n}=F_{n, 1}+\cdots+F_{n, L}$. Clearly $F_{n}$ is $\left(\Gamma_{n}, \varepsilon / 2\right)$-spanning for the subgroup

$$
H_{n}=\sum_{j=1}^{L} \operatorname{Fix}_{\Delta_{n, j}}(\sigma) \subset \operatorname{Fix}_{\Gamma_{n}}(\sigma) \subset \mathbb{T}^{\mathbb{Z}^{d}}
$$

and

$$
\left|F_{n}\right| \leqslant \prod_{j=1}^{L}\left|F_{n, j}\right| \leqslant \prod_{j=1}^{L}\left(\frac{4 L}{\varepsilon}\right)^{\left|\Omega_{\Gamma_{n}} \cap H_{\mathbf{m}_{j}}\right|} \leqslant\left(\frac{4 L}{\varepsilon}\right)^{\frac{L M\left|\Omega_{\Gamma_{n}}\right|}{\left\langle\Gamma_{n}\right\rangle}} .
$$

Since $\left|\mathbb{Z}^{d} / \Gamma_{n}\right|=\left|\Omega_{\Gamma_{n}}\right|$ and $\left\langle\Gamma_{n}\right\rangle \rightarrow \infty$, it follows that

$$
\lim _{n \rightarrow \infty} \frac{1}{\left|\mathbb{Z}^{d} / \Gamma_{n}\right|} \log \left|F_{n}\right|=0 .
$$

Since the cardinality of every $\left(\Gamma_{n}, \varepsilon\right)$-separated set is bounded by the cardinality of every $\left(\Gamma_{n}, \varepsilon / 2\right)$-spanning set, every sequence of $\left(\Gamma_{n}, \varepsilon\right)$-separated sets $F_{n}^{\prime} \subset H_{n}$ must also satisfy that

$$
\lim _{n \rightarrow \infty} \frac{1}{\left|\mathbb{Z}^{d} / \Gamma_{n}\right|} \log \left|F_{n}^{\prime}\right|=0 .
$$

Choose the finite set $A_{\varepsilon}$ according to Lemma 6.2. Let $\left(Q_{n}\right)_{n \geqslant 1}$ be a Følner sequence of fundamental domains for the groups $\left(\Gamma_{n}\right)_{n \geqslant 1}$, and put $Q_{n}^{\prime}=\bigcap_{\mathbf{m} \in A_{\varepsilon}}\left(Q_{n}-\right.$ $\mathbf{m})$. Then by Lemma 6.3, we have that $\left(Q_{n}^{\prime}\right)_{n \geqslant 1}$ is again a Følner sequence with 
$\left|Q_{n}^{\prime}\right| /\left|Q_{n}\right| \rightarrow 1$ as $n \rightarrow \infty$. From Lemma 6.4 we know that $\operatorname{Fix}_{\Gamma_{n}}\left(\alpha_{f}\right)$ contains a $\left(\Gamma_{n}, \varepsilon\right)$-separated set $F_{n}(\varepsilon) \subset X_{f}$ which is $\left(Q_{n}^{\prime}, 2 \varepsilon\right)$-spans $X_{f}$.

For $n \geqslant 1$, the intersection of $F_{n}(\varepsilon)$ with every coset of the group $H_{n}$ in $\operatorname{Fix}_{\Gamma_{n}}(\sigma)$ is $\left(\Gamma_{n}, \varepsilon\right)$-separated. We set

$$
D_{n}(\varepsilon)=\max _{y \in \mathrm{Fix}_{\Gamma_{n}}(\sigma)}\left|F_{n}(\varepsilon) \cap\left(y+H_{n}\right)\right| \geqslant \max _{y \in \mathrm{Fix}_{\Gamma_{n}}(\sigma)}\left|F_{n}(\varepsilon) \cap\left(y+\operatorname{Fix}_{\Gamma_{n}}^{\circ}\left(\alpha_{f}\right)\right)\right|
$$

and conclude from (6.2) that

$$
\lim _{n \rightarrow \infty} \frac{1}{\left|\mathbb{Z}^{d} / \Gamma_{n}\right|} \log D_{n}(\varepsilon)=0 .
$$

Up to now $\varepsilon$ was fixed, but now we start varying it. For every $n \geqslant 1, F_{n}(\varepsilon)$ has to intersect at least $\left|F_{n}(\varepsilon)\right| / D_{n}(\varepsilon)$ distinct cosets of Fix $\Gamma_{\Gamma_{n}}^{\circ}\left(\alpha_{f}\right)$. By (6.1) and (6.3),

$$
\begin{aligned}
\liminf _{n \rightarrow \infty} \frac{1}{\left|\mathbb{Z}^{d} / \Gamma_{n}\right|} & \log \left|\operatorname{Fix}_{\Gamma_{n}}\left(\alpha_{f}\right) / \operatorname{Fix}_{\Gamma_{n}}^{\circ}\left(\alpha_{f}\right)\right| \\
& \geqslant \sup _{\varepsilon>0} \liminf _{n \rightarrow \infty} \frac{1}{\left|\mathbb{Z}^{d} / \Gamma_{n}\right|} \log \left(\left|F_{n}(\varepsilon)\right| / D_{n}(\varepsilon)\right) \\
& =\sup _{\varepsilon>0} \liminf _{n \rightarrow \infty} \frac{1}{\left|\mathbb{Z}^{d} / \Gamma_{n}\right|} \log \left|F_{n}(\varepsilon)\right|=\mathrm{h}\left(\alpha_{f}\right) .
\end{aligned}
$$

Combining this with the previously mentioned fact that $\mathrm{p}^{+}\left(\alpha_{f}\right)=\mathrm{h}\left(\alpha_{f}\right)$ completes the proof of Theorem 1.3 .

\section{Diophantine consequences}

If $f \in R_{d}$ is atoral, then Theorem 1.3 shows that the Riemann sums for $\log |f|$ over $\Omega_{\Gamma}$ converge to its integral $\mathrm{m}(f)$ as $\langle\Gamma\rangle \rightarrow \infty$. Roughly speaking, this means that those $\boldsymbol{\omega} \in \Omega_{\Gamma}$ lying close to $\mathrm{U}(f)$ cannot contribute a disproportionate amount to the Riemann sum, and this prevents these $\boldsymbol{\omega}$ from getting very close to $\boldsymbol{U}(f)$.

When $d=1$ this phenomenon was first established by Gelfond [9] using quite different methods. He showed that if $\lambda \in \mathbb{S}$ is an algebraic number, then for all $\varepsilon>0$ the inequality

$$
\left|\lambda^{n}-1\right|>e^{-\varepsilon n}
$$

holds for all sufficiently large $n$. This can be reformulated as follows. For $f \in R_{1}$ with $\mathrm{U}(f) \neq \varnothing$, for all sufficiently large $n$ the inequality

$$
\operatorname{dist}\left(\mathrm{U}(f), \Omega_{n \mathbb{Z}}\right)>e^{-\varepsilon\left|\Omega_{n \mathbb{Z}}\right|}
$$

holds. When $d \geqslant 2$ our method gives a result formally identical to this. However, it of course does not apply when $d=1$ since for this case $f$ cannot be atoral unless $\mathrm{U}(f)=\varnothing$.

For $0 \neq f \in R_{d}$ let us say that the Riemann sums for $\log |f|$ converge to $\mathrm{m}(f)$ provided that

$$
\frac{1}{\left|\Omega_{\Gamma}\right|} \sum_{\boldsymbol{\omega} \in \Omega_{\Gamma} \backslash \mathrm{U}(f)} \log |f(\boldsymbol{\omega})| \rightarrow \mathrm{m}(f):=\int_{\mathbb{S}^{d}} \log |f(\mathbf{s})| d \lambda(\mathbf{s}) \quad \text { as }\langle\Gamma\rangle \rightarrow \infty .
$$


By Theorem 1.3, this is valid for all atoral Laurent polynomials.

We begin by establishing a quantitative consequence of the convergence of the Riemann sums.

Lemma 7.1. Let $0 \neq f \in R_{d}$, and assume that the Riemann sums for $\log |f|$ converge to $\mathrm{m}(f)$. Let $r_{n}>0$ and $\Gamma_{n} \in \mathcal{F}$ such that $r_{n} \rightarrow 0$ and $\left\langle\Gamma_{n}\right\rangle \rightarrow \infty$ as $n \rightarrow \infty$. Then

$$
\frac{1}{\left|\Omega_{\Gamma_{n}}\right|} \sum_{\substack{\boldsymbol{\omega} \in \Omega_{\Gamma_{n}} \\ 0<|f(\boldsymbol{\omega})|<r_{n}}}|\log | f(\boldsymbol{\omega})|| \rightarrow 0 \quad \text { as } n \rightarrow \infty
$$

Proof. Let $\varepsilon>0$. For $r>0$ define $\phi_{r}(\mathbf{s}):=\max \{|f(\mathbf{s})|, r\}$ and $E_{r}(f):=\left\{\mathbf{s} \in \mathbb{S}^{d}\right.$ : $0<|f(\mathbf{s})|<r\}$. We may assume throughout that $r_{n}, r<1$, so that $\log |f(\boldsymbol{\omega})|<0$ for all $\boldsymbol{\omega} \in E_{r}(f)$.

Note that $\log \phi_{r}$ is continuous on $\mathbb{S}^{d}$, and so is integrable there. Furthermore, $\log |f| \in L^{1}\left(\mathbb{S}^{d}, \lambda\right)$, and $\log \phi_{r} \searrow \log |f|$. By the Monotone Convergence Theorem, we can find $r_{0}>0$ such that

$$
0 \leqslant \int_{\mathbb{S}^{d}} \log \phi_{r} d \lambda-\int_{\mathbb{S}^{d}} \log |f| d \lambda<\varepsilon
$$

for all $0<r<r_{0}$. We may also choose $r_{0}$ small enough so that $\lambda\left(E_{r_{0}}(f)\right)<\varepsilon$.

By our assumption about convergence of Riemann sums, we have that

$$
\left|\mathrm{m}(f)-\frac{1}{\left|\Omega_{\Gamma}\right|} \sum_{\boldsymbol{\omega} \in \Omega_{\Gamma} \backslash \mathrm{U}(f)} \log \right| f(\boldsymbol{\omega})||<\varepsilon
$$

for $\langle\Gamma\rangle$ sufficiently large. Since $\log \phi_{r_{0}}$ is continuous we also have that

$$
\left|\int \log \phi_{r_{0}} d \lambda-\frac{1}{\left|\Omega_{\Gamma}\right|} \sum_{\boldsymbol{\omega} \in \Omega_{\Gamma}} \log \right| \phi_{r_{0}}(\boldsymbol{\omega})||<\varepsilon
$$

for sufficiently large $\langle\Gamma\rangle$. Finally, a standard argument using upper and lower approximations of the indicator of function of $E_{r_{0}}(f)$ by continuous functions shows that

$$
\frac{\left|E_{r_{0}}(f) \cap \Omega_{\Gamma}\right|}{\left|\Omega_{\Gamma}\right|}<\varepsilon
$$

for all sufficiently large $\langle\Gamma\rangle$. Thus we may choose $L_{0}$ large enough so that (17.4), (7.5), and (7.6) hold if $\langle\Gamma\rangle>L_{0}$.

Now assume that $r_{n} \rightarrow 0$ and $\left\langle\Gamma_{n}\right\rangle \rightarrow \infty$. Choose $n_{0}$ such that $r_{n}<r_{0}$ and $\left\langle\Gamma_{n}\right\rangle>L_{0}$ for all $n \geqslant n_{0}$. Then since $E_{r_{n}}(f) \subset E_{r_{0}}(f)$ and $\log f(\mathbf{s})<0$ for all 
$\mathbf{s} \in E_{r_{0}}(f)$, it follows that

$$
\begin{aligned}
& 0 \leqslant \frac{1}{\left|\Omega_{\Gamma_{n}}\right|} \sum_{\boldsymbol{\omega} \in \Omega_{\Gamma_{n}} \cap E_{r_{n}}(f)}|\log | f(\boldsymbol{\omega})|| \leqslant \frac{1}{\left|\Omega_{\Gamma_{n}}\right|} \sum_{\boldsymbol{\omega} \in \Omega_{\Gamma_{n}} \cap E_{r_{0}}(f)}-\log |f(\boldsymbol{\omega})| \\
& =\frac{1}{\left|\Omega_{\Gamma_{n}}\right|} \sum_{\boldsymbol{\omega} \in \Omega_{\Gamma_{n}}} \log \phi_{r_{0}}(\boldsymbol{\omega})-\frac{\left(\log r_{0}\right)\left|\Omega_{\Gamma_{n}} \cap E_{r_{0}}(f)\right|}{\left|\Omega_{\Gamma_{n}}\right|}-\sum_{\boldsymbol{\omega} \in \Omega_{\Gamma_{n}} \backslash \mathrm{U}(f)} \log |f(\boldsymbol{\omega})| \\
& \leqslant \mathrm{m}\left(\phi_{r_{0}}\right)+\varepsilon-\varepsilon \log r_{0}-(\mathrm{m}(f)-\varepsilon)<3 \varepsilon+\varepsilon \log r_{0} .
\end{aligned}
$$

Since $\varepsilon>0$ was arbitrary, this completes the proof.

To make use of this result, we introduce two counting functions. For $r>0$ let

$$
M_{f}\left(\Omega_{\Gamma}, r\right):=\left|\left\{\boldsymbol{\omega} \in \Omega_{\Gamma}: 0<\operatorname{dist}(\boldsymbol{\omega}, \mathrm{U}(f))<r\right\}\right|,
$$

and

$$
N_{f}\left(\Omega_{\Gamma}, r\right):=\left|\left\{\boldsymbol{\omega} \in \Omega_{\Gamma}: 0<|f(\boldsymbol{\omega})|<r\right\}\right| .
$$

Observe that $f$ is Lipschitz on $\mathbb{S}^{d}$, say with Lipschitz constant $K$. Then $M_{f}\left(\Omega_{\Gamma}, r\right) \leqslant$ $N_{f}\left(\Omega_{\Gamma}, K r\right)$.

Theorem 7.2. Let $0 \neq f \in R_{d}$, and assume that the Riemann sums for $\log |f|$ converge to $\mathrm{m}(f)$. Let $r_{n} \rightarrow 0$ and $\left\langle\Gamma_{n}\right\rangle \rightarrow \infty$ as $n \rightarrow \infty$. Then

$$
\frac{M_{f}\left(\Omega_{\Gamma_{n}}, r_{n}\right) \cdot \log \left(1 / r_{n}\right)}{\left|\Omega_{\Gamma_{n}}\right|} \rightarrow 0 \text { as } n \rightarrow \infty \text {. }
$$

In particular, for every $\varepsilon>0$ there is an $n_{0}$ such that

$$
M_{f}\left(\Omega_{\Gamma_{n}}, r_{n}\right)<\frac{\varepsilon\left|\Omega_{\Gamma_{n}}\right|}{\log \left(1 / r_{n}\right)} \text { for } n \geqslant n_{0} .
$$

Proof. Let $\varepsilon>0$, and $K \geqslant 1$ be a Lipschitz constant for $f$ on $\mathbb{S}^{d}$. By Lemma [7.1, for all sufficiently large $n$ we have that

$$
\frac{1}{\left|\Omega_{\Gamma_{n}}\right|} N_{f}\left(\Omega_{\Gamma_{n}}, K r_{n}\right) \log \frac{1}{r_{n}} \leqslant \frac{1}{\left|\Omega_{\Gamma_{n}}\right|} \sum_{\substack{\boldsymbol{\omega} \in \Omega_{\Gamma_{n}} \\ 0<|f(\boldsymbol{\omega})|<K r_{n}}}|\log | f(\boldsymbol{\omega})||<\varepsilon .
$$

Since $M_{f}\left(\Omega_{\Gamma_{n}}, r_{n}\right) \leqslant N_{f}\left(\Omega_{\Gamma_{n}}, L r_{n}\right)$ and $\log \left(1 / L r_{n}\right) \leqslant \log \left(1 / r_{n}\right)$, the result follows.

Corollary 7.3. Let $f \in R_{d}$ be an atoral Laurent polynomial and $\varepsilon>0$. Then

$$
\operatorname{dist}\left(\Omega_{\Gamma}, \mathrm{U}(f) \backslash \Omega_{\Gamma}\right)>e^{-\varepsilon\left|\Omega_{\Gamma}\right|}
$$

whenever $\langle\Gamma\rangle$ is sufficiently large.

Proof. Suppose there is an $\delta_{0}>0$ and a sequence $\Gamma_{n} \in \mathcal{F}$ with $\left\langle\Gamma_{n}\right\rangle \rightarrow \infty$ such that

$$
\operatorname{dist}\left(\Omega_{\Gamma_{n}}, \mathrm{U}(f) \backslash \Omega_{\Gamma_{n}}\right)<e^{-\delta_{0}\left|\Omega_{\Gamma_{n}}\right|}
$$

for all $n \geqslant 1$. Put $r_{n}=e^{-\delta_{0}\left|\Omega_{\Gamma_{n}}\right|} \rightarrow 0$. By the previous theorem, with $\varepsilon=\delta_{0} / 2$, for sufficiently large $n$ we would have

$$
1 \leqslant M_{f}\left(\Omega_{\Gamma_{n}}, r_{n}\right)<\frac{\delta_{0}}{2} \frac{\left|\Omega_{\Gamma_{n}}\right|}{\delta_{0}\left|\Omega_{\Gamma_{n}}\right|}=\frac{1}{2}
$$


which is impossible.

We can use Theorem $[7.2$ to obtain slightly weaker results even when $f$ is toral (e.g., in Gelfond's original setting). Let $\mathbb{Z}_{+}$denote $\{0,1,2, \ldots\}$.

Theorem 7.4. Let $d \geqslant 1$ and $f \in R_{d}$ be a nonzero irreducible Laurent polynomial. For every $\varepsilon>0$ and every $\psi: \mathbb{Z}_{+} \rightarrow \mathbb{Z}_{+}$with $\psi(n) \rightarrow \infty$ as $n \rightarrow \infty$ (no matter how slowly), there is an $L \geqslant 1$ such that for every $\Gamma \in \mathcal{F}$ with $\langle\Gamma\rangle>L$ and every $\boldsymbol{\omega} \in \Omega_{\Gamma} \backslash \mathrm{U}(f)$ we have that

$$
\operatorname{dist}(\boldsymbol{\omega}, \mathrm{U}(f)) \geqslant e^{-\varepsilon \psi(\langle\Gamma\rangle)\left|\Omega_{\Gamma}\right|} .
$$

Proof. Let $g(u)=u-1$, and consider the Laurent polynomial

$$
h\left(u_{1}, \ldots, u_{d}, u_{d+1}\right)=f\left(u_{1}, \ldots, u_{d}\right) f^{*}\left(u_{1}, \ldots, u_{d}\right)+g\left(u_{d+1}\right) g^{*}\left(u_{d+1}\right) \in R_{d+1} .
$$

Clearly $\mathrm{U}(h)=\mathrm{U}(f) \times\{1\}$, and so $h$ is atoral.

Let $\psi: \mathbb{Z}_{+} \rightarrow \mathbb{Z}_{+}$with $\psi(n) \rightarrow \infty$ as $n \rightarrow \infty$. For every finite index subgroup $\Gamma$ of $\mathbb{Z}^{d}$ and every $k \geqslant 1$, let $\Gamma^{(k)}$ be the finite-index subgroup of $\mathbb{Z}^{d+1}$ generated by $\Gamma \times\{0\}$ and $(\mathbf{0}, \psi(k))$. Then $\left|\mathbb{Z}^{d+1} / \Gamma^{(k)}\right|=\left|\mathbb{Z}^{d} / \Gamma\right| \psi(k)$ and $\left\langle\Gamma^{(k)}\right\rangle=\min \{\langle\Gamma\rangle, \psi(k)\}$. We can then apply Corollary 7.3 to obtain (7.7).

Applying the previous theorem when $d=1$, we obtain the following weaker version of Gelfond's result.

Corollary 7.5. Let $\gamma \in \mathbb{S}$ be an algebraic number which is not a root of unity. Then for every $\psi: \mathbb{Z}_{+} \rightarrow \mathbb{Z}_{+}$with $\psi(n) \rightarrow \infty$ as $n \rightarrow \infty$ and every $\varepsilon>0$, there is a $n_{0}$ such that for every $n \geqslant n_{0}$ and every nth root of unity $\omega \in \Omega_{n \mathbb{Z}}$ we have that

$$
\operatorname{dist}(\gamma, \omega) \geqslant e^{-\varepsilon \psi(n) n} \text {. }
$$

Remark 7.6. In Corollary 7.5 we allow $\psi(n)$ to grow arbitrarily slowly. But we still require $\psi(n) \rightarrow \infty$, so that this is not quite strong enough to obtain Gelfond's result (7.1). Thus this also does not imply the convergence of the logarithmic growth rate of periodic points for quasihyperbolic toral automorphisms. For this one really needs (7.1).

\section{REFERENCES}

[1] J. Agler, J.E. McCarthy, and M. Stankus, Toral algebraic sets and function theory on polydisks, J. Geom. Anal. 16 (2006), no. 4, 551-562.

[2] Iskander Aliev and Chris Smyth, Solving algebraic equations in roots of unity, preprint, 2010.

[3] Saugata Basu, Richard Pollack, Marie-Françoise Roy, Algorithms in Real Algebraic Geometry (2nd ed.), Springer-Verlag, New York, 2009.

[4] David Boyd, Kronecker's theorem and Lehmer's problem for polynomials in several variables, J. Number Theory 13 (1981), 116-121.

[5] C. Deninger, Fuglede-Kadison determinants and entropy for actions of discrete amenable groups, J. Amer. Math. Soc. 19 (2006), 737-758.

[6] C. Deninger and K. Schmidt, Expansive algebraic actions of discrete residually finite amenable groups and their entropy, Ergod. Th. \& Dynam. Sys. 27 (2007), 769-786.

[7] Lou van den Dries, Tame topology and O-minimal Structures, Cambridge University Press, Cambridge, 1998.

[8] Harold M. Edwards, Divisor Theory, Birkhäuser, Boston, 1990. 
[9] A.O. Gelfond, Transcendental and Algebraic Numbers, Dover, New York, 1960.

[10] A. Granville and Z. Rudnick, Torsion points on curves, NATO Sci. Ser. II Math. Phys. Chem. 237 (2007), 85-92.

[11] D. Lind, Dynamical properties of quasihyperbolic toral automorphisms, Ergodic Theory \& Dyn. Sys. 2 (1982), 49-68.

[12] D. Lind and K. Schmidt, Homoclinic points of algebraic $\mathbf{Z}^{d}$-actions, J. Amer. Math. Soc. 12 (1999), 953-980.

[13] D. Lind, K. Schmidt and E. Verbitskiy, Entropy and growth rate of periodic points of algebraic $\mathbf{Z}^{d}$-actions, in: Dynamical Numbers: Interplay between Dynamical Systems and Number Theory, ed. S. Kolyada, Yu. Manin, M. Möller, P. Moree and T. Ward, Contemp. Math., vol. 532, American Mathematical Society, Providence, R.I., 2010.

[14] D. Lind, K. Schmidt and T. Ward, Mahler measure and entropy for commuting automorphisms of compact groups, Invent. Math. 101 (1990), 593-629.

[15] H.B. Mann, On linear relations between roots of unity, Mathematika 12 (1965), 107-117.

[16] T. Schick, $L^{2}$-determinant class and approximation of $L^{2}$-Betti numbers, Trans. Amer. Math. Soc. 353 (2001), 3247-3265.

[17] K. Schmidt, Automorphisms of compact abelian groups and affine varieties, Proc. London Math. Soc. 61 (1990), 480-496.

[18] K. Schmidt, Dynamical Systems of Algebraic Origin, Birkhäuser Verlag, Basel-Berlin-Boston, 1995.

[19] K. Schmidt and E. Verbitskiy, Abelian sandpiles and the harmonic model, Comm. Math. Phys. 292 (2009), 721-759.

[20] C.J. Smyth, An explicit formula for the Mahler measure of a family of 3-variable polynomials, Journal de Théorie de Nombres de Bordeaux 14 (2002), 683-700.

[21] E. Stein, Harmonic Analysis: Real Variable Methods, Orthogonality, and Oscillatory Integrals, Princeton Univ. Press, Princeton, 1993.

Douglas Lind: Department of Mathematics, University of Washington, Seattle, WASHINGTON 98195, USA

E-mail address: lind@math.washington.edu

Klaus Schmidt: Mathematics Institute, University of Vienna, Nordbergstrasse 15, A-1090 Vienna, Austria

E-mail address: klaus.schmidt@univie.ac.at

Evgeny Verbitskiy: Mathematical Institute, University of Leiden, PO Box 9512, 2300 RA LEIDEN, The Netherlands

and

Johann Bernoulli Institute for Mathematics and Computer Science, University of Groningen, PO Box 407, 9700 AK, Groningen, The Netherlands

E-mail address: evgeny@math.leidenuniv.nl 